\title{
A Study of the Nambu-Jona-Lasinio Model on the Lattice
}

\author{
Khalil M. Bitar and Pavlos M. Vranas \\ Supercomputer Computations Research Institute \\ The Florida State University \\ Tallahassee, FL 32306
}

May 27, 2022

\begin{abstract}
We present our full analysis of the two flavor Nambu-Jona-Lasinio model with $S U(2) \times S U(2)$ chiral symmetry on the four-dimensional hypercubic lattice with naive and Wilson fermions. We find that this model is an excellent toy field theory to investigate issues related to lattice QCD. We use the large $N$ approximation to leading order in $1 / N$ to obtain non perturbative analytical results over almost the whole parameter range. By using numerical simulations we estimate that the size of the $1 / N$ corrections for most of the quantities we consider are small and in this way we strengthen the validity of the leading order large $N$ calculations. We obtain results regarding the approach to the continuum chiral limit, the effects of the zero momentum fermionic modes on finite lattices and the scalar and pseudoscalar spectrum.
\end{abstract}

PACS 12.40.-y, 11.30.Rd, 12.38.Gc 


\section{Introduction}

At present the low energy properties of QCD can only be studied numerically using lattice gauge theory. It is believed that in order to obtain reasonable results in reasonable time periods the computing power needed is of the order of one Teraflop. Although supercomputers with such capacity may be built in the next few years it is still important to use other methods to get insights into the physics as well as into the behavior of the theory on the lattice.

The Nambu-Jona-Lasinio (NJL) model was introduced before the discovery of quarks as a theory of nucleons interacting with a four-Fermi interaction. Today the fermionic fields of the model are reinterpreted as being those of the quarks. The most important feature of the model that qualifies it to describe some of the important low energy properties of QCD is that it possesses the same chiral symmetry as QCD and that this symmetry can be realized in the Goldstone mode.

The NJL model can also be motivated by an argument found in [1]. The gauge field in the full theory of QCD develops a finite correlation length of the size of the inverse mass of the lightest glueball ( $\approx 1550 \mathrm{MeV}$, see for example [2]). If we consider QCD on a lattice and integrate the high momentum fluctuations of the fermionic and gauge fields down to $\Lambda \lesssim 1550 \mathrm{MeV}$ then the lattice spacing will be of the order of the correlation length and we must then essentially have a theory of fermions with contact interactions and cutoff $\Lambda$. The resulting effective Lagrangian will maintain the original chiral symmetry but will of course be more complicated. If we further restrict our attention to energies much below the cutoff, naively speaking, it should be enough to keep in the Lagrangian the least irrelevant operator namely the four-Fermi dimension six operator. This is the NJL model.

Unfortunately, by only keeping the four-Fermi operator, valuable information was lost and the model does not confine the quarks. Therefore, strictly speaking, it can not be a true effective field theory of QCD. Furthermore, if, for example, we want to study the $\sigma$ particle, which on phenomenological grounds is believed to have mass $\approx 750 \mathrm{MeV}$, then the separation of scales is probably not large enough to justify the neglect of operators with dimension higher than six. Nevertheless, as mentioned above, the NJL model possesses the same chiral symmetry as QCD and it can realize this symmetry in the Goldstone mode. It is this feature that is most crucial in the understanding of the lightest hadrons and the reason for the successful quantitative predictions of the model.

The NJL model has been studied extensively for various cases with continuum type regularizations. For a comprehensive review the reader is referred to [3] and references therein. Also for some recent work in the continuum see [4]. Furthermore the NJL model is a special case of Yukawa models that, under a different context, have been studied extensively with lattice regularization [5]. The model has also been studied on the lattice [6] in connection with the possible equivalence of the top quark condensate with the Higgs field [7]. In that work, however, the separation of scales is very large (the cutoff is of the order $10^{14} \mathrm{GeV}$ ), and it is therefore quite a different problem than the one considered here.

In this paper we do not attempt to use the NJL model to make physical predictions. Our interest in the NJL model is purely qualitative and originates from the fact that

its lattice version is an excellent toy model to investigate issues related to lattice QCD. This is the case primarily because it is an effective four dimensional fermionic theory that 
has the same chiral symmetry as QCD, which symmetry in an appropriate phase may be realized in the Goldstone mode, and because one can use the large $N$ approximation to leading order in $1 / N$ to obtain non perturbative analytical results over almost the whole parameter range. Also by using numerical simulations of the model one can estimate the size of the $1 / N$ corrections and in this way strengthen the validity of the leading order large $N$ calculations. As it will be shown in this paper the $1 / N$ corrections estimated in this way are small for most of the quantities we consider. This means that one has a good analytical handle on the model. Because of this a wealth of information can be extracted.

For naive fermions, we calculate the scalar and pseudoscalar spectrum as a function of the cutoff and find agreement with general expectations. This is not perceived as a quantitative prediction but rather as a first approximation to a physical picture. Furthermore, since the NJL model possesses the right symmetry of a possible embedding theory of the Higgs Sector it can therefore provide an interesting example of such a theory. We briefly discuss this possibility.

One of the main purposes of our work involves the study of the Wilson formulation of lattice fermions. This formulation breaks the chiral symmetry of the model explicitly. The symmetry is expected to be restored as a result of the tuning of the Wilson parameter $\kappa$ to some critical value $\kappa_{c}$. Needless to say this is a very important issue for QCD and it would be very useful to clarify how it is realized in a simpler model. This involves, for example, issues such as what the continuum chiral limit on a lattice is and how does one define $\kappa_{c}$. For Wilson fermions we also determine the effect of the heavy doublers on the sigma particle. We show that the heavy doublers raise the mass of the sigma to be of order cutoff.

Another issue investigated in this paper involves the study of the effects of the zero modes on finite lattices. This is of particular importance to numerical simulations. We demonstrate that in certain cases these effects are large and can obscure the extrapolation to infinite volume physics. We also estimate the size of the $1 / N$ corrections by comparing leading order large $N$ results on finite size lattices with numerical results on the same size lattices and find that in most cases the $1 / N$ corrections are small. This is of interest since, as mentioned above, the NJL model has been and is studied extensively as a phenomenological model using continuum type regularizations and among other methods the leading order large $N$ approximation.

In this paper we consider the two flavor (up and down) NJL model with $S U(2) \times S U(2)$ chiral symmetry and $\mathrm{SU}(\mathrm{N})$ color symmetry, with scalar and pseudoscalar couplings [8] on the four dimensional hypercubic lattice. We consider both naive and Wilson fermions and we study the model using a large $N$ expansion as well as a Hybrid Monte Carlo (HMC) numerical simulation. A shorter version of this work containing only the main results has appeared elsewhere [9].

Throughout the paper we use a notation whereby small letters denote quantities in lattice units and capital letters denote quantities in physical ( $\mathrm{MeV}$ or $\mathrm{GeV}$ ) units. The lattice spacing is denoted by " $a$ ".

The paper is organized as follows. The model and its lattice version are described in Section 2. The large $N$ analysis to leading order in large $N$ is given in Section 3. Using asymptotic expansions we study in Section 3.1 the case of naive fermions on an infinite lattice and present results regarding the scalar-pseudoscalar sector of QCD and briefly 
discuss their possible relevance to the Higgs sector. In Section 3.2 we study the case of Wilson fermions on an infinite lattice using asymptotic expansions and present results regarding the approach to the chiral continuum limit as well as the effect of the doublers on the scalar particle self energy. The numerical and large $N$ work on finite lattices is described in Section 4. We present there, in Section 4.1, a comparison of our numerical results with the large $N$ results obtained on same size lattices and obtain an estimate of the size of the $1 / N$ corrections. In Section 4.2 we examine the effects of the zero momentum quark modes on finite lattices in connection to the inversion time of the Conjugate Gradient algorithm used in the HMC, and more importantly in connection to finite size effects. Furthermore after the preprint version of this work appeared in the computer list "hep-lat" and during the refereeing process of this paper another group [10] presented complementary results. In particular, for the case of Wilson fermions, the authors showed, using the leading order large $N$ approximation, that there is a phase where the remaining flavor-parity symmetry breaks spontaneously generating a non zero vacuum expectation value for the pion field. Their work was done at infinite volume using numerical integration and among other things it was shown that the phase line is a line at which all three pions are massless. This line was plotted for all effective quark masses. In this work we always stay on the symmetric side of this line where the vacuum expectation value of the pion field is zero. At infinite volume, using asymptotic expansions, we had calculated the part of this line (fig. 2a) that corresponds to small effective quark masses since this is the region where continuum physics is extracted. Our calculations, up to errors relevant to the approximations used, are in agreement. However, on a finite volume the comparison is not as straight forward and it deserves special attention. For this reason section 4.3 was added where the zero pion mass line on a finite volume is discussed in detail. Finally, a short summary and conclusion is given in Section 5.

\section{The model}

The Lagrangian density in Minkowski space and in continuum notation is:

$$
\mathcal{L}=\bar{\Psi}\left(i \not \partial-m_{0}\right) \Psi+\frac{G_{1}}{2}\left[(\bar{\Psi} \Psi)^{2}+\left(\bar{\Psi} i \gamma_{5} \tau \Psi\right)^{2}\right]
$$

In the above expression all indices have been suppressed. The fermionic field $\Psi$ is a flavor $S U(2)$ doublet and a color $S U(N) N$-column vector. The Lagrangian is diagonal in color, in contrast with the full QCD Lagrangian which is diagonal in flavor. $\boldsymbol{\tau}=\left\{\tau_{1}, \tau_{2}, \tau_{3}\right\}$ are the three isospin Pauli matrices, $\not \partial=\gamma^{\mu} \partial_{\mu}$, and $m_{0}$ is the bare quark mass (if $m_{0} \neq 0$ the chiral symmetry is explicitly broken). To obtain a Lagrangian that is quadratic in

the fermionic fields we introduce the scalar auxiliary field $\sigma$ and the three pseudoscalar auxiliary fields $\boldsymbol{\pi}=\left\{\pi_{1}, \pi_{2}, \pi_{3}\right\}$. Using the functional identity [8]

$$
\begin{aligned}
& \exp \left\{i \frac{G_{1}}{2} \int d^{4} x\left[(\bar{\Psi} \Psi)^{2}+\left(\bar{\Psi} i \gamma_{5} \boldsymbol{\tau} \Psi\right)^{2}\right]\right\} \sim \\
& \int[d \sigma d \boldsymbol{\pi}] \exp \left\{i \int d^{4} x\left[-\bar{\Psi}\left(\sigma+i \gamma_{5} \boldsymbol{\tau} \cdot \boldsymbol{\pi}\right) \Psi-n_{f} \beta_{1}\left(\sigma^{2}+\boldsymbol{\pi}^{2}\right)\right]\right\}
\end{aligned}
$$


the Lagrangian density becomes

$$
\begin{aligned}
\mathcal{L} & =\bar{\Psi} M \Psi-n_{f} \beta_{1}\left(\sigma^{2}+\boldsymbol{\pi}^{2}\right) \\
M & =i \not \partial-m_{0}-\sigma-i \gamma_{5} \boldsymbol{\tau} \cdot \boldsymbol{\pi} .
\end{aligned}
$$

Here $n_{f}=2$ is the number of flavors and $\beta_{1}=\frac{1}{2 n_{f} G_{1}}$. The fermionic fields can now be integrated and the resulting partition function is

$$
Z=\int[d \sigma d \boldsymbol{\pi}][\operatorname{det} M]^{N} e^{-i n_{f} \beta_{1} \int d^{4} x\left(\sigma^{2}+\boldsymbol{\pi}^{2}\right)} .
$$

Notice that there is no explicit kinetic energy term for the $\sigma$ and $\boldsymbol{\pi}$ fields. As we will show soon this term is part of $\operatorname{det} M$.

In the general case the fermionic determinant has a phase that is related to the the chiral anomaly and the Wess-Zumino term [11]. In our work because the flavor space is restricted to $S U(2)$ the phase is not present and we will write our action in a symmetric fashion with regards to $M$ and $M^{\dagger}$. Going to Euclidean space and appropriately discretizing the above Lagrangian we obtain the model on the Euclidean Hypercubic lattice. On the lattice, as it is well known, we have species doubling. The doubling in the NJL model will be interpreted as a doubling of the color degrees of freedom. To treat this problem we add to the Lagrangian density an irrelevant operator (Wilson term) of the form $\frac{a r}{2} \bar{\Psi} \partial^{2} \Psi$, " $a$ " being the lattice spacing and " $r$ " a constant. We consider the $r=0$ case where no effort is made to remove the doublers (naive fermions) and also the $r \neq 0$ case where the doubler masses are raised to the cutoff (Wilson fermions) and the chiral symmetry is explicitly broken. With these considerations and after appropriate scaling of the fields and couplings, so that only dimensionless quantities appear, we obtain:

$$
\begin{aligned}
Z & =\int[d \Psi d \bar{\Psi} d \sigma d \boldsymbol{\pi}] e^{-S} \\
S & =\sum_{x, y}\left\{\sum_{i=1}^{N / 2}\left\{\bar{\Psi}_{x}^{i} M_{x y} \Psi_{y}^{i}+\bar{\Psi}_{x}^{i+N / 2} M_{x y}^{\dagger} \Psi_{y}^{i+N / 2}\right\}+n_{f} \beta_{1}\left(\sigma_{x}^{2}+\boldsymbol{\pi}_{x}^{2}\right) \delta_{x y}\right\} \\
M_{x y} & =\frac{1}{2} \sum_{\mu}\left[\left(\gamma_{\mu}-r\right) \delta_{x+\mu, y}-\left(\gamma_{\mu}+r\right) \delta_{x-\mu, y}\right]+\left(4 r+m_{0}+\sigma_{x}+i \gamma_{5} \boldsymbol{\pi}_{\boldsymbol{x}} \cdot \boldsymbol{\tau}\right) \delta_{x y}
\end{aligned}
$$

with $\gamma_{\mu}$ hermitian. This partition function can be cast into two different forms. The first can be studied using a large $N$ expansion and is given by:

$$
\begin{aligned}
Z & =\int[d \sigma d \boldsymbol{\pi}] e^{-S_{1}} \\
S_{1} & =N\left\{-\frac{1}{2} \operatorname{Tr}(\log M)-\frac{1}{2} \operatorname{Tr}\left(\log M^{\dagger}\right)+n_{f} \tilde{\beta}_{1} \sum_{x}\left(\sigma_{x}^{2}+\boldsymbol{\pi}_{x}^{2}\right)\right\}
\end{aligned}
$$

with $\tilde{\beta}_{1}=\beta_{1} / N$ and the trace taken over space, spin, flavor, and color. The second is appropriate for numerical simulations and is given by:

$$
\begin{aligned}
Z & =\int\left[d X d X^{\dagger} d \sigma d \boldsymbol{\pi}\right] e^{-S_{2}} \\
S_{2} & =\sum_{x, y}\left\{\sum_{i=1}^{N / 2}\left\{X_{x}^{i \dagger}\left(M^{\dagger} M\right)_{x y}^{-1} X_{y}^{i}\right\}+n_{f} \beta_{1}\left(\sigma_{x}^{2}+\pi_{y}^{2}\right) \delta_{x y}\right\}
\end{aligned}
$$

with $X$ being pseudofermionic fields. 


\section{Large $N$}

We perform a standard large $N$ expansion with the action of eq. 6. The large $N$ approximation will be reasonable for as long as $\tilde{\beta}_{1}$ is of order one. We assume a translation invariant saddle and small fluctuations around it. For the case of naive fermions using the chiral symmetry we rotate the fields so that the saddle field configuration lies along the $\sigma$ direction. For the case of Wilson fermions the chiral symmetry is broken and the saddle field configuration lies along the $\sigma$ direction. As was shown in [10, the remaining parityflavor symmetry can spontaneously break generating a non zero vacuum expectation value for the pion field. However, in this work we will always stay in the parity-flavor symmetric phase where the pion field has zero expectation value. We have:

$$
\sigma(x)=\sigma_{s}+\frac{\delta \sigma(x)}{\sqrt{N}}, \quad \boldsymbol{\pi}(x)=\frac{\delta \boldsymbol{\pi}(x)}{\sqrt{N}}
$$

In momentum space the inverse quark propagator at the saddle is:

$$
\tilde{M}_{s}(p)=i \sum_{\mu} \gamma_{\mu} \sin p_{\mu}+r\left(4-\sum_{\mu} \cos p_{\mu}\right)+m_{0}+\sigma_{s}
$$

We identify

$$
m_{0}+\sigma_{s}=m_{q}
$$

as the quark mass. This definition is valid close to the continuum limit where $m_{q}$ is small. It must be pointed out that $m_{q}$ is the constituent quark mass since all high energy gluonic degrees of freedom of QCD have been integrated out. In physical units the quark mass will be taken to be equal to one third the proton mass.

We expand around the saddle (expansion in $\left.\frac{\delta \sigma(x)}{\sqrt{N}}, \frac{\delta \boldsymbol{\pi}(x)}{\sqrt{N}}\right)$. The zero order gives the effective potential

$$
\frac{V_{e f f}}{N}=-2 n_{f} \int_{p} \log \left[g\left(p, m_{q}\right)\right]+n_{f} \tilde{\beta}_{1} \sigma_{s}^{2}
$$

where $\int_{p}=\int_{p \in B} \frac{d^{4} k}{(2 \pi)^{4}}$ for an infinite lattice (B denotes the hypercubic lattice Brillouin zone) and $\int_{p}=\frac{1}{L_{x}^{3} L_{t}} \sum_{\left\{n_{1}, n_{2}, n_{3}, n_{4}\right\}}$ with $n_{1}, n_{2}, n_{3} \in\left[0, L_{x}-1\right], n_{4} \in\left[0, L_{t}-1\right]$ and $p=$ $\left(\frac{2 \pi n_{1}}{L_{x}}, \frac{2 \pi n_{2}}{L_{x}}, \frac{2 \pi n_{3}}{L_{x}}, \frac{2 \pi n_{4}}{L_{t}}\right)$ for a finite lattice with spatial extend $L_{x}$ and temporal extent $L_{t}$. In the above equation $g\left(p, m_{q}\right)$ is defined as

$$
\begin{aligned}
g\left(p, m_{q}\right) & =\sum_{\nu} \sin ^{2} p_{\nu}+\left[r w(p)+m_{q}\right]^{2}=p^{2}+m_{q}^{2}+O\left(p^{2} m_{q}\right) \\
w(p) & =4-\sum_{\mu} \cos p_{\mu}=\frac{p^{2}}{2}+O\left(p^{4}\right) .
\end{aligned}
$$

The saddle point equations are obtained at the point where the linear term vanishes.

$$
\sigma_{s} \frac{\tilde{\beta}_{1}}{2}-\int_{p} \frac{\sigma_{s}+m_{0}+r w(p)}{g\left(p, m_{0}+\sigma_{s}\right)}=0
$$


The $\sigma$ and $\boldsymbol{\pi}$ propagators $G_{\sigma}(q)$ and $G_{\pi}(q)$ can be obtained from the second order term of the expansion around the saddle $\frac{1}{2} \int_{q} \delta \sigma(q) G_{\sigma}^{-1} \delta \sigma(-q)+\frac{1}{2} \int_{q} \delta \boldsymbol{\pi}(q) G_{\pi}^{-1} \delta \boldsymbol{\pi}(-q)$ with:

$$
\begin{aligned}
G_{\pi / \sigma}^{-1}(q) & =4 n_{f}\left[\frac{\tilde{\beta}_{1}}{2}-I_{\pi / \sigma}(q)\right] \\
I_{\pi / \sigma}(q) & =\int_{k} \frac{\sum_{\nu} \sin \left(k_{\nu}+\frac{q_{\nu}}{2}\right) \sin \left(k_{\nu}-\frac{q_{\nu}}{2}\right)+/-\left[r w\left(k+\frac{q}{2}\right)+m_{q}\right]\left[r w\left(k-\frac{q}{2}\right)+m_{q}\right]}{g\left(k+\frac{q}{2}, m_{q}\right) g\left(k-\frac{q}{2}, m_{q}\right)}
\end{aligned}
$$

We define the pion wave function renormalization constant $Z_{\pi}$ and pion mass $m_{\pi}$ from:

$$
\lim _{q \rightarrow 0} G_{\pi}^{-1}(q)=Z_{\pi}^{-1}\left(q^{2}+m_{\pi}^{2}\right)
$$

With this definition we find using eq. 14 and 15:

$$
\begin{aligned}
Z_{\pi}^{-1} & =\frac{n_{f}}{2} \int_{k} \frac{\sum_{\mu}\left[\cos ^{2} k_{\mu}+r^{2} \sin ^{2} k_{\mu}\right]}{g\left(k, m_{q}\right)^{2}} \\
Z_{\pi}^{-1} m_{\pi}^{2} & =4 n_{f}\left\{\frac{\tilde{\beta} 1}{2}-\int_{k} \frac{1}{g\left(k, m_{q}\right)}\right\} .
\end{aligned}
$$

Notice that $Z_{\pi}$ is positive and therefore does not create local stability problems (the propagator is positive). Also notice that with this definition of the pion mass, $m_{\pi}$ will be the true pole of $G_{\pi}$ only if $m_{\pi}$ is small. For the cases we are interested in this will always be true. Unfortunately we can not define the $\sigma$ mass $m_{\sigma}$ and width $\gamma_{\sigma}$ in a similar way since $m_{\sigma}$ will not be small in general. The proper definition in this case is:

$$
G_{\sigma}^{-1}\left(q=\left\{i m_{\sigma}+\frac{\gamma_{\sigma}}{2}, 0,0,0\right\}\right)=0
$$

and we can not obtain closed form expressions for $m_{\sigma}$ and $\gamma_{\sigma}$.

On a finite lattice the finite momentum sums can be calculated with the aid of a computer and the above quantities except $\gamma_{\sigma}$ can be exactly determined. This will be done in Section 4.1 when we will compare the numerical results obtained on a finite lattice with the large $N$ predictions for the same lattice size. For an infinite lattice the momentum integrals can be evaluated numerically but this will not serve our purposes since we are interested in obtaining analytical expressions. For this reason we resort into performing asymptotic expansions in small $m_{q}, m_{\pi}, m_{\sigma}$, and $q$, considering the logarithms as being of order zero. The asymptotic expansions will be given separately for the $r=0$ and $r \neq 0$ cases in the next two sections.

\subsection{Naive fermions at infinite volume, $r=0$}

In this case we have species doubling. The doubling is interpreted as a "doubling" of the color degrees of freedom. That this interpretation is appropriate can be seen from eq. 11. For $r=0$ the function $g\left(p, m_{q}\right)$ of eq. 12 is periodic with period $\pi$ and not $2 \pi$. This means 
that the integral in eq. 11 splits into 16 equal pieces and therefore the effective potential will be made up from $16 N$ copies. Therefore we set the number of colors $N_{c}$ to:

$$
N_{c}=16 N
$$

Some of the typical integrals we will encounter are:

$$
\begin{aligned}
J_{n}\left(m_{q}\right) & =\int_{k} \frac{1}{g\left(k, m_{q}\right)^{n}} \\
I_{0}\left(q, m_{q}\right) & =\int_{k} \frac{1}{g\left(k+\frac{q}{2}, m_{q}\right) g\left(k-\frac{q}{2}, m_{q}\right)} .
\end{aligned}
$$

Simple trigonometric relations relate these integrals with the corresponding integrals that arise for Bose particles. We get

$$
\begin{aligned}
J_{n}(m) & =16\left\{4^{n-2} J_{n}^{B}(2 m)\right\} \\
I_{0}(q, m) & =16 I_{0}^{B}(2 q, 2 m)
\end{aligned}
$$

where $J_{n}^{B}$ and $I_{0}^{B}$ are defined as in eq. 21 but with $g(k, m)=2 \sum_{\nu}\left[1-\cos \left(k_{\nu}\right)\right]+m^{2}$ the inverse propagator of a Bosonic particle. The asymptotic expansions of $J_{1}^{B}$ and $J_{2}^{B}$ have been evaluated to leading order in $m_{q}$ with very accurately determined coefficients in [12 Appendix B. The leading order term of $I_{0}^{B}$ is universal except for a lattice constant. For example, it has been evaluated for the $F_{4}$ lattice in [13]. The lattice constant needed also appears in the leading order term of the asymptotic expansion of $J_{2}^{B}$ and for the hypercubic lattice can be taken from [12]. For the convenience of the reader we present these results below:

$$
\begin{aligned}
J_{1}^{B}(m) & =r_{0}+m^{2}\left(r_{1}+s_{1} \log m^{2}\right)+O(4) \\
J_{n+1}^{B}(m) & =-\frac{1}{n} \frac{\partial}{\partial m^{2}} J_{n}^{B}(m) \\
I_{0}^{B}(q, m) & =-s_{1} \int_{0}^{1} d s \log \left[m^{2}+s(1-s) q^{2}\right]-\left(r_{1}+s_{1}\right)+O(2)
\end{aligned}
$$

with

$$
\begin{aligned}
r_{0} & =0.154933390 \\
r_{1} & =-0.030345755 \\
s_{1} & =\frac{1}{16 \pi^{2}} .
\end{aligned}
$$

Because $r=0$, if we set $m_{0}=0$ the chiral symmetry is not explicitly broken. In that case there are two solutions to the saddle point equation [13, namely $\sigma_{s}=0$ and $\sigma_{s} \neq 0$. For $\tilde{\beta}_{1}>\tilde{\beta}_{1_{c}}$, the dominating saddle is $\sigma_{s}=0$ and we are in a chirally symmetric phase with massive $\sigma$ and $\boldsymbol{\pi}$ fields and massless quarks $m_{q}=\sigma_{s}=0$. For $\tilde{\beta}_{1}<\tilde{\beta}_{1_{c}}$ the dominating saddle is $\sigma_{s} \neq 0$ and we are in a phase with spontaneously broken chiral symmetry. The pions are the Goldstone bosons and become massless. The sigma is the massive mode and the quarks acquire a dynamically generated mass $m_{q}=\sigma_{s} \neq 0$. The critical value of $\tilde{\beta}_{1}$ is:

$$
\tilde{\beta}_{1_{c}}=2 J_{1}(0)=8 r_{0} .
$$


All of our analysis will be done in the broken phase.

The asymptotic expansion of $G_{\pi}^{-1}(q)$ is:

$$
G_{\pi}^{-1}(q)=Z_{\pi}^{-1} m_{\pi}^{2}+4 n_{f} q^{2}\left\{\frac{1}{2} I_{0}\left(q, m_{q}\right)-\frac{1}{8} J_{1}(0)\right\}+O(4)
$$

where only the leading order term of $Z_{\pi}^{-1} m_{\pi}^{2}$ and $I_{0}$ is to be kept. The coefficient of the $q^{2}$ term will be equal to $Z_{\pi}$ for $q \rightarrow 0$. The pion mass mass should satisfy $G_{\pi}^{-1}(q=$ $\left.\left\{i m_{\pi}, 0,0,0\right\}\right)=0$. To this order in the asymptotic expansion this will be true only if the coefficient of the $q^{2}$ term for $q=\left\{i m_{\pi}, 0,0,0\right\}$ is very close to $Z_{\pi}$. From the asymptotic expansion of $I_{0}\left(q, m_{q}\right)$ (eq. 22, 23) we see that this will happen for $m_{\pi}^{2} s(1-s) \ll m_{q}^{2}$ where $s \in[0,1]$. Therefore for this definition of $m_{\pi}$ to be valid we must not only demand that $m_{\pi}$ is small, as mentioned in the previous section, but also that:

$$
m_{\pi}^{2} \ll 4 m_{q}^{2}
$$

For all the cases we will be interested in this will be satisfied. For example with $M_{q}=$ $310 \mathrm{MeV}$ and $M_{\pi}=140 \mathrm{MeV}$ we get $\frac{M_{\pi}^{2}}{4 M_{q}^{2}}=0.054$.

Equation 17 can be rewritten in the form:

$$
Z_{\pi}^{-1}=\frac{n_{f}}{2}\left[\left(4+m_{q}^{2}\right) J_{2}\left(m_{q}\right)-J_{1}\left(m_{q}\right)\right] .
$$

Using eq. 22, 23 and 28 we find the leading order term of the asymptotic expansion of $Z_{\pi}$ :

$$
\begin{aligned}
Z_{\pi}^{-1} & =16\left[z_{0}-z_{1} \log m_{q}^{2}\right] \\
z_{0} & =-2 n_{f}\left(\frac{r_{0}}{16}+r_{1}+s_{1}+s_{1} \log 4\right), \text { for } n_{f}=2 \quad z_{0}=0.022204130 \\
z_{1} & =2 n_{f} s_{1} .
\end{aligned}
$$

As mentioned in the previous section $Z_{\pi}$ is always positive and does not create local stability problems. From the above equation we find that $Z_{\pi}$ will become negative if $m_{q}>\exp \frac{z_{0}}{2 z_{1}} \simeq$ 1.55. This provides us with a point above which the small $m_{q}$ approximation is certainly not valid. In our analysis we will never need to be close to this point. However, the above equation has a much more important consequence. It can be shown [8] that the pion decay constant $f_{\pi}$ is related to $m_{q}$ and $Z_{\pi}$ via:

$$
f_{\pi}^{2}=N m_{q}^{2} Z_{\pi}^{-1}
$$

Using eq. 30, 29 and 20 we get:

$$
\frac{f_{\pi}^{2}}{m_{q}^{2}}=N_{c}\left[z_{0}-z_{1} \log m_{q}^{2}\right]
$$

Then with $N_{c}=3, n_{f}=2, \frac{f_{\pi}}{m_{q}}=\frac{F_{\pi}}{M_{q}}=\frac{93 \mathrm{MeV}}{310 \mathrm{MeV}}=0.3$ and with $m_{q}=M_{q} a, \Lambda=\frac{\pi}{a}$ we find $\Lambda=1150 \mathrm{MeV}$. This is consistent with the expectation that the cutoff of the theory should be $\lesssim 1550 \mathrm{MeV}$ (the mass of the lightest glueball [2]). 
Although, as mentioned in the introduction, it is not the aim of this work to study the possibility of the Higgs being a top quark condensate, we point out that similar considerations as in the previous paragraph can be used to estimate the cutoff of such a theory. With $N_{c}=3, n_{f}=1, F_{\pi}=246 \mathrm{GeV}, M_{q}=170 \mathrm{GeV}$ we obtain $\Lambda=3 \times 10^{14} \mathrm{GeV}$. This is consistent with the much more complete and detailed analysis of [0]].

Equation 18 can be rewritten in the form:

$$
m_{\pi}^{2}=4 n_{f} Z_{\pi} \frac{m_{0}}{m_{q}-m_{0}} J_{1}\left(m_{q}\right)
$$

where the saddle point equation 13 was used to eliminate $\tilde{\beta}_{1}$. Using eq. 32, 23 and 22, we find the leading order term of the asymptotic expansion of $m_{\pi}$ :

$$
m_{\pi}^{2}=\frac{m_{0}}{\left(m_{q}-m_{0}\right)} \frac{n_{f} r_{0}}{\left[z_{0}-z_{1} \log m_{q}^{2}\right]}+O(2)
$$

Notice that when the chiral symmetry is not explicitly broken, namely $m_{0}=0$, then in the broken phase where $m_{q}-m_{0}=\sigma_{s} \neq 0, m_{\pi}=0$ as it should.

Next we concentrate on $m_{\sigma}$ and $\gamma_{\sigma}$. Using the saddle point equation 13 to eliminate $\tilde{\beta}_{1}$ and eq. 29 the leading order term of the asymptotic expansion of the inverse sigma propagator of eq. 14 is:

$$
\frac{G_{\sigma}^{-1}(q)}{16}=\frac{M_{\pi}^{2}}{F_{\pi}^{2}} N_{c}\left[z_{0}-z_{1} \log m_{q}^{2}\right]^{2} m_{q}^{2}+4 n_{f}\left\{\frac{1}{2}\left(q^{2}+4 m_{q}^{2}\right) I_{0}^{B}\left(2 q, 2 m_{q}\right)-\frac{r_{0}}{32} q^{2}\right\}+O(4)
$$

where only the leading order term of $I_{0}^{B}$ given in eq. 23 is to be kept. The leading order term of $I_{0}^{B}$ contains a $\log (-1)$ that we take to be equal to $+i \pi$ (taking it to be equal to $-i \pi$ results to a negative width). Using the above equation and the definition 19 we can calculate $m_{\sigma}$ and $\gamma_{\sigma}$. Because we can not get a closed form expression we solve numerically and the result is shown in Figure 1.

From Figure 1 we find that if we set the quark mass to one third the proton mass $M_{q}=310 \mathrm{MeV}$ then $M_{\sigma}=726 \mathrm{MeV}, \Gamma_{\sigma}=135 \mathrm{MeV}$, and $\Lambda=\pi / a=1150 \mathrm{MeV} . M_{\sigma}$ is consistent with phenomenological expectations and as mentioned earlier $\Lambda$ is consistent with the expectation that the cutoff should be close and below the mass of the lightest glueball $(1550 \mathrm{MeV})$. The width however is underestimated. The reason is traced to the fact that to leading order in large $N$ the width receives contributions only from the quark bubble and not from the pion bubble because the pion bubble is of order $1 / N$. Because the phase space available for the $\sigma$ to decay to two quarks is much smaller than the phase space to decay to two pions, the pion loop contribution, although of order $1 / N$, is probably more important than the quark loop contribution.

The above result can also be used to make an interesting observation. If the Higgs sector is the low energy effective field theory of a NJL model with exactly the same parameters as the low energy QCD except for $M_{\pi}=0$ and $F_{\pi}=246 \mathrm{GeV}$, then we find $M_{\sigma}=$ $1915 \mathrm{GeV}$. This corresponds to $m_{\sigma}=2$ where one would expect very large deviations from the low energy behavior of scattering cross sections. Although we have not calculated these deviations the value of the width serves as an indication of their size. In a way, departure 
from low energy behavior will be signaled by an increasing width of the $\sigma$ to two quark decay. At $m_{\sigma} \approx 2$ the width is already fairly large.

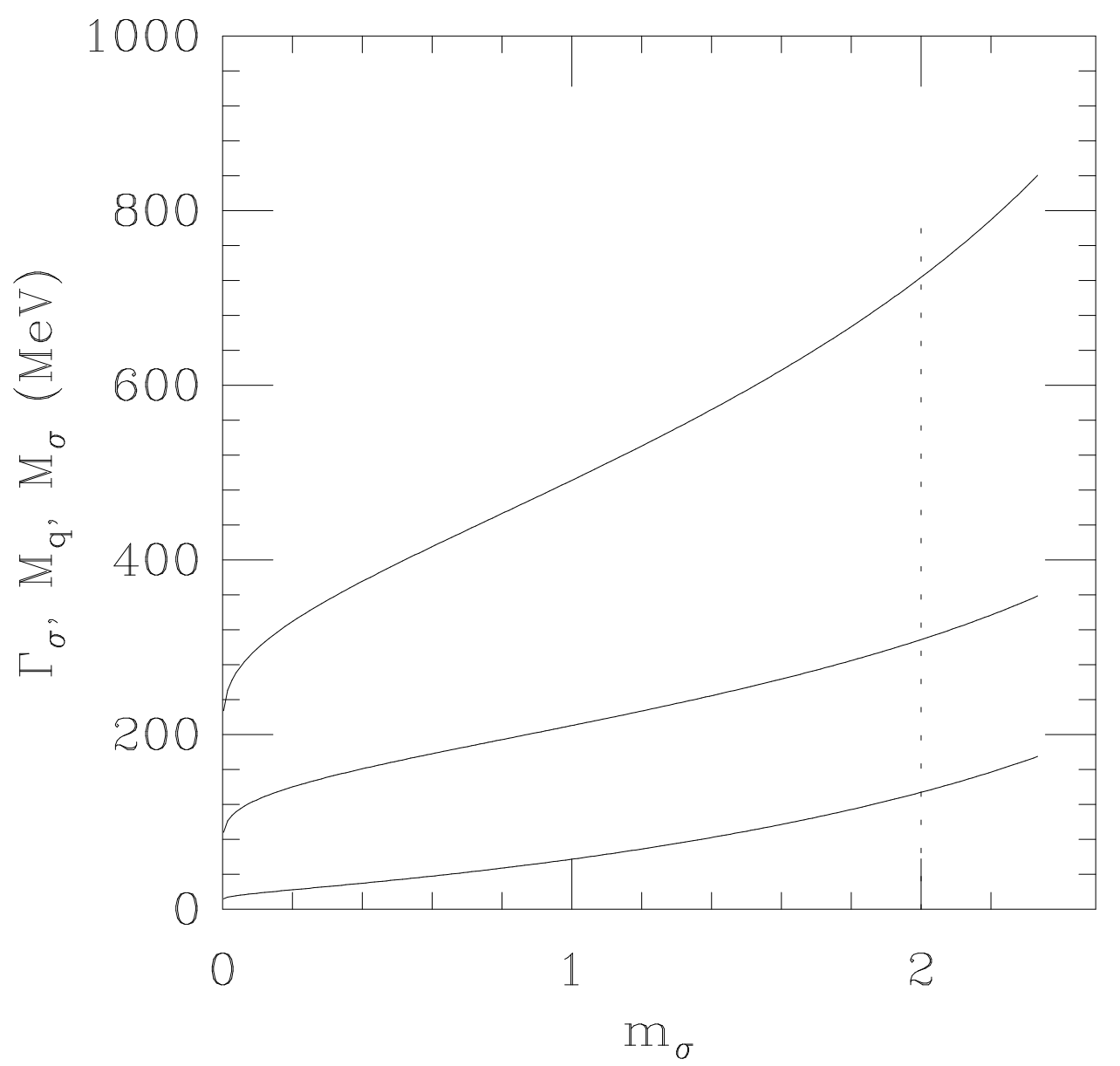

Figure 1. $r=0, N_{c}=3, F_{\pi}=93 \mathrm{MeV}$, and $M_{\pi}=140 \mathrm{MeV}$. From top to bottom the lines correspond to the $\sigma$ mass, quark mass and $\sigma$ width calculated in the large $N$ approximation to leading order in $m_{q}$. The vertical line denotes the point where the quark mass is equal to $310 \mathrm{MeV}$.

As a final comment notice that close to the continuum limit and by setting the renormalization point of the integral $I_{0}^{B}$ in eq. 34 at $q=0$ we obtain

$$
\begin{aligned}
m_{\sigma}^{2} & =m_{\pi}^{2}+4 m_{q}^{2} \\
\gamma_{\sigma} & =0
\end{aligned}
$$

where we have used the definition of eq. 19. This is the result obtained in the literature with continuum type regularizations (see for example [8]). This result, because of the off-- 
shell renormalization point, is only approximate and also neglects the contribution of the quarks to $\gamma_{\sigma}$.

\subsection{Wilson fermions at infinite volume, $r \neq 0$}

In this case the doublers have been removed by raising their masses to the cutoff. The chiral symmetry has been explicitly broken by the Wilson term and the pions are massive. The number of colors is:

$$
N_{c}=N
$$

Some of the typical integrals we will encounter are:

$$
\begin{aligned}
J_{n, m}\left(m_{q}\right) & =\int_{k} \frac{w(k)^{n}}{g\left(k, m_{q}\right)^{m}} \\
I_{0}\left(q, m_{q}\right) & =\int_{k} \frac{1}{g\left(k+\frac{q}{2}, m_{q}\right) g\left(k-\frac{q}{2}, m_{q}\right)} .
\end{aligned}
$$

Unfortunately there are no simple trigonometric relations that relate these integrals with the corresponding integrals that arise for Bose particles as in the $r=0$ case. We will need the asymptotic expansions of $J_{0,1}, J_{1,1}$ to order $m_{q}^{2}$ and the leading order term of $J_{0,2}$. We have calculated them and the result is given in Appendix A. We will also need the leading order asymptotic expansion of the $I_{0}$ integral. This, up to the lattice constant $r_{1}$ that needs to be calculated and is given in Appendix A, is the same as the leading order term of $I_{0}^{B}$ in eq. 23. Some of the lattice constants that we will need can be parametrized as:

$$
a_{n, m}=J_{n, m}(0) \quad m-n \leq 1
$$

In our analysis only few of them appear and we have calculated them for $r=1$ and $r=0.1$. They are given in Appendix A together with the constants

$$
\begin{aligned}
r_{1} & =\int_{k}\left\{\frac{1}{g(k, 0)^{2}}-\frac{\Theta\left(l^{2}-k^{2}\right)}{k^{4}}\right\}+\frac{1}{16 \pi^{2}} \log \left(l^{2}\right) \\
s_{1} & =\frac{1}{16 \pi^{2}} .
\end{aligned}
$$

where $\Theta$ is the step function.

The asymptotic expansion of $G_{\pi}^{-1}(q)$ is:

$$
G_{\pi}^{-1}(q)=Z_{\pi}^{-1} m_{\pi}^{2}+4 n_{f} q^{2}\left\{\frac{1}{2} I_{0}\left(q, m_{q}\right)+\frac{\left(r^{2}-1\right)}{8} a_{0,1}-\frac{r^{2}\left(r^{2}-1\right)}{8} a_{2,2}\right\}+O(3)
$$

where only the leading order term of $Z_{\pi}^{-1} m_{\pi}^{2}$ and $I_{0}$ is to be kept. The same restrictions on $m_{\pi}$ as in eq. 27 are needed.

The leading order term of the asymptotic expansion of $Z_{\pi}$ is:

$$
\begin{aligned}
Z_{\pi}^{-1} & =\left[z_{0}-z_{1} \log m_{q}^{2}\right] \\
z_{0} & =-2 n_{f}\left[r_{1}+s_{1}+\frac{1-r^{2}}{4} a_{0,1}-\frac{r^{2}\left(1-r^{2}\right)}{4} a_{2,2}\right], \text { for } n_{f}=2, r=1, \quad z_{0}=0.0223 \\
z_{1} & =2 n_{f} s_{1} .
\end{aligned}
$$


Notice that for $r=1, z_{0}$ has a value that is very close to the one of the $r=0$ case. Because of that and since $z_{1}$ is universal all the discussion relating to eq. 29, 30 and 31 is also valid here.

Equation 18 can be rewritten in the form:

$$
m_{\pi}^{2}=4 n_{f} Z_{\pi} \frac{1}{m_{q}-m_{0}}\left[m_{0} J_{0,1}\left(m_{q}\right)+r J_{1,1}\left(m_{q}\right)\right]
$$

where the saddle point equation 13 was used to eliminate $\tilde{\beta}_{1}$. The leading order term of the asymptotic expansion of $m_{\pi}$ is:

$$
m_{\pi}^{2}=\frac{4 n_{f}}{\left(m_{q}-m_{0}\right)} \frac{m_{0} a_{0,1}+r a_{1,1}}{\left[z_{0}-z_{1} \log m_{q}^{2}\right]}+O(2) .
$$

We are now in a position to investigate the approach to the continuum chiral limit. The theory has two adjustable bare parameters, $\beta_{1}$ and $m_{0}$. The parameter $m_{0}$ is related to the more familiar hoping parameter $\kappa$ (often used in QCD) by:

$$
\kappa=\frac{1}{8 r+2 m_{0}}
$$

The two bare parameters control $m_{q}$ and $m_{\pi}$ through equations 13 and 42 . The following statements can be made:

A The $m_{q}=0$ line where the continuum limit is retrieved is defined by:

$$
\beta_{1}+\frac{2 N_{c} r a_{1,1}}{m_{0}}=0
$$

B For any $m_{q}, m_{0}$ can be adjusted so that $m_{\pi}=0$. The $m_{\pi}=0$ line is given by:

$$
m_{0} J_{0,1}\left(m_{q}\right)+r J_{1,1}\left(m_{q}\right)=0
$$

As was shown in [10] this line separates the flavor-parity symmetric phase from the flavorparity broken phase. In this work we always stay in the symmetric phase.

C The continuum chiral limit is obtained when both $m_{q}$ and $m_{\pi}$ go to zero. This point is:

$$
m_{0_{\text {chiral }}}=-r \frac{a_{1,1}}{a_{0,1}}, \quad \beta_{1_{\text {chiral }}}=-\frac{2 N_{c} r a_{1,1}}{m_{0}}
$$

and all physics should be extracted at the vicinity of this point.

Using the asymptotic expansions of eq. 13 and 42 to order $m_{q}^{2}$, we plot for small $m_{q}$ and $m_{\pi}$ the constant $m_{q}$ and constant $m_{\pi}$ lines for $r=1$ in Figure $2 \mathrm{a}$ and for $r=0.1$ in Figure 2b. The two Figures are qualitatively the same with the $m_{q}=0, m_{\pi}=0$ point shifted toward larger $\kappa$ and $\beta_{1}$. In fact as $r \rightarrow 0$ it can be easily shown that $\beta_{1} \rightarrow N \tilde{\beta}_{1_{c}}$, where $\tilde{\beta}_{1_{c}}$ is the critical value of the $r=0$ case (see eq. 25), and $\kappa \rightarrow \infty$. From eq. 43 we find that as $m_{q} \rightarrow 0$ also $m_{\pi} \sim 1 / \log m_{q}^{2} \rightarrow 0$. This implies that the $m_{q}=0$ line is also an $m_{\pi}=0$ line. This behavior is apparent in Figure 2a and 2b. However, this is an artifact of our approximation in defining $m_{\pi}$. As mentioned earlier (see the comment after 
eq. 40) our definition of $m_{\pi}$ will be valid only if $m_{\pi}^{2} \ll 4 m_{q}^{2}$ which can not be satisfied on the $m_{q}=0$ line except on the one point where it intersects the $m_{\pi}=0$ line. The constant $m_{\pi}$ lines are therefore valid only in the regions where $m_{\pi}^{2} \ll 4 m_{q}^{2}$. To get the full constant $m_{\pi}$ lines a more detailed analysis would be necessary

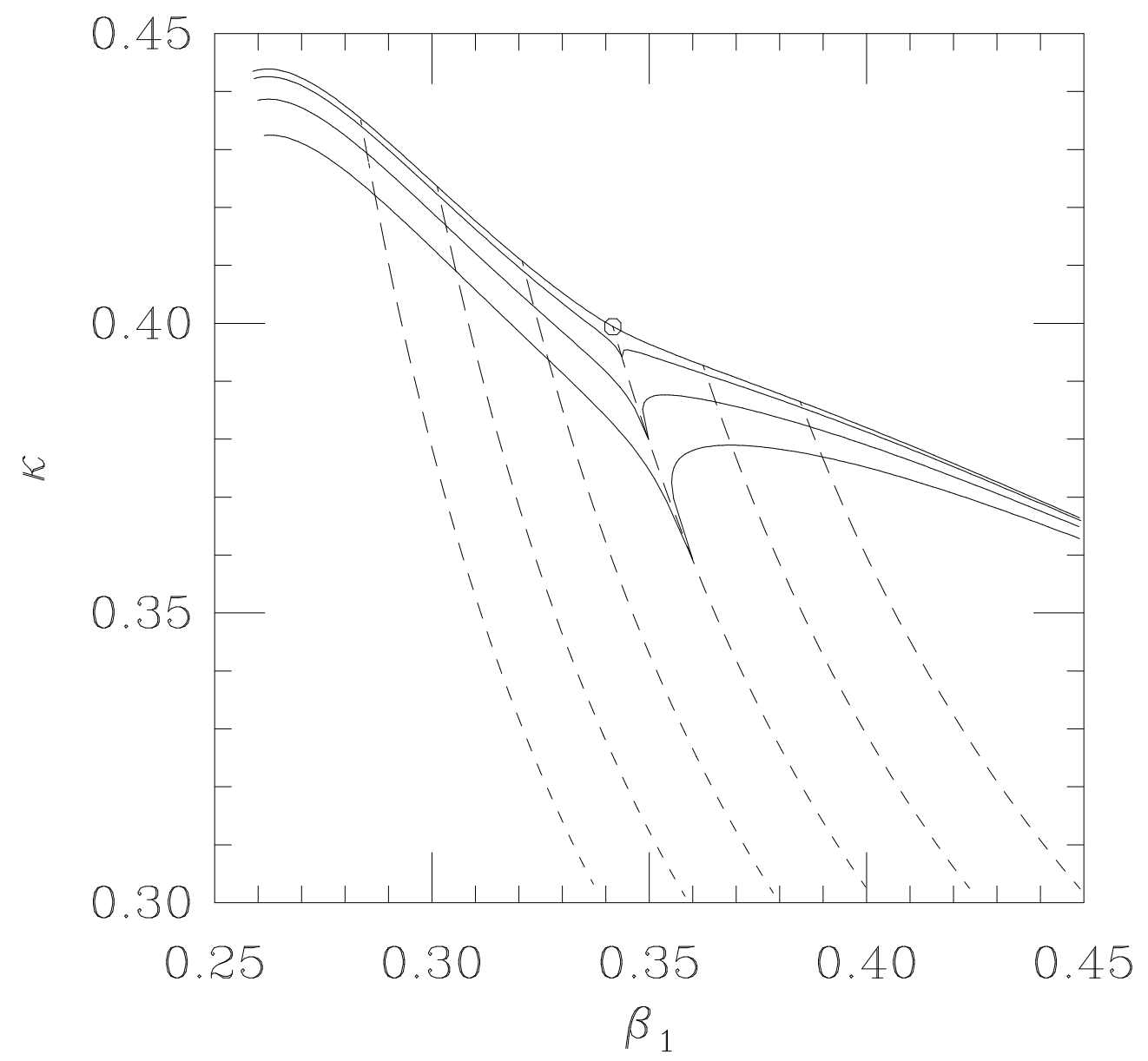

Figure 2a. The $\beta_{1}, \kappa=1 /\left(8+2 m_{0}\right)$ plane for $r=1.0, N_{c}=2$. The solid lines are constant $m_{\pi}$ lines. From top to bottom they correspond to $m_{\pi}=0,0.1,0.2,0.3$. The dotted lines are constant $m_{q}$ lines. From right to left they correspond to $m_{q}=-0.2,-0.1,0.0,0.1,0.2$, 0.3. The $m_{q}=0, m_{\pi}=0$ point is located at $\beta_{1_{\text {chiral }}}=0.3416, \kappa_{\text {chiral }}=0.3994$.

From these Figures we see that if for a fixed $\beta_{1}$ we were to change $\kappa$ from smaller to larger values (as is often done in QCD with dynamical Wilson fermions) then if $\beta_{1}<\beta_{1_{\text {chiral }}}$ we would reach the $m_{\pi}=0$ limit before we reach the continuum limit. On the other hand if $\beta_{1}>\beta_{1_{\text {chiral }}}$ we would reach the continuum limit before we reach the $m_{\pi}=0$ limit. As mentioned in "C" above, there is only one point in the $\beta_{1}, \kappa$ plane where we can obtain a 
continuum chiral limit.

Using the saddle point equation 13 the chiral condensate is found to be:

$$
\frac{\langle\bar{\Psi} \Psi\rangle}{n_{f}}=-2 \tilde{\beta}_{1}\left(m_{q}-m_{0}\right)=-2 \tilde{\beta}_{1} \sigma_{s}
$$

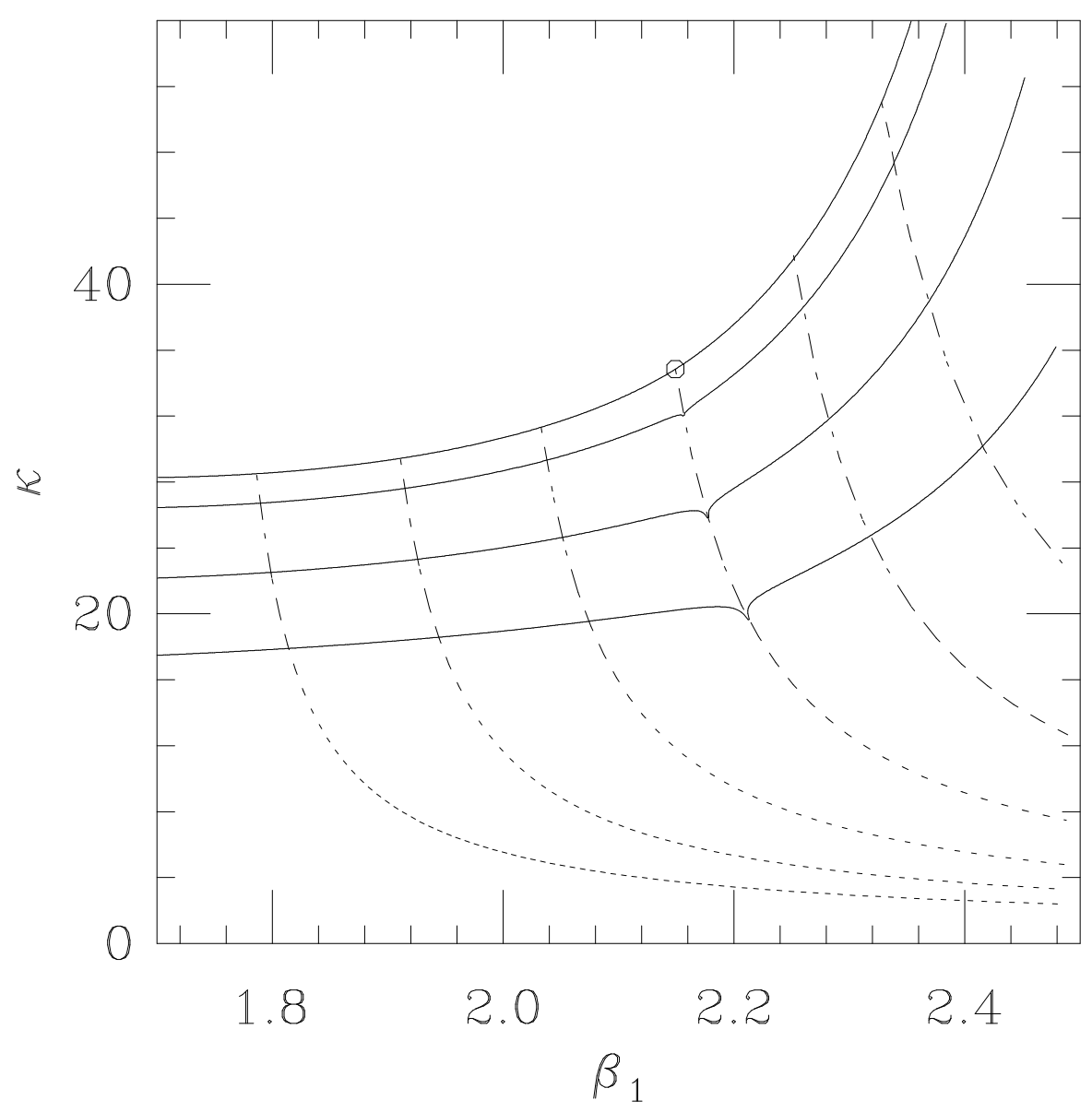

Figure 2b. Same as in Figure 2a but for $r=0.1$. The $m_{q}=0, m_{\pi}=0$ point is located at $\beta_{1}=2.1492, \kappa=34.843$.

and it is not zero when $m_{q}=0$ and/or $m_{\pi}=0$. Therefore it can not serve as an order parameter for Wilson fermions. On the other hand when $r=0$ and $m_{0}=0\langle\bar{\Psi} \Psi\rangle$ is an order parameter because $\sigma_{s}$ is an order parameter.

Next we concentrate on $m_{\sigma}$ and $\gamma_{\sigma}$. The leading order term of the asymptotic expansion 
of the inverse sigma propagator of eq. 14 is:

$$
\begin{aligned}
G_{\sigma}^{-1}(q) & =\frac{M_{\pi}^{2}}{F_{\pi}^{2}} N_{c}\left[z_{0}-z_{1} \log m_{q}^{2}\right]^{2} m_{q}^{2} \\
& +4 n_{f}\left\{\frac{1}{2}\left(q^{2}+4 m_{q}^{2}\right) I_{0}\left(q, m_{q}\right)+q^{2} \frac{\left(r^{2}-1\right)}{8} a_{0,1}+R_{0}+m_{q} R_{1}+m_{q}^{2} R_{2}+q^{2} R_{3}\right\} \\
& +O(3)
\end{aligned}
$$

where only the leading order term of $I_{0}$ is to be kept. $R_{0}, R_{1}, R_{2}$, and $R_{3}$ are lattice constants defined below.

$$
\begin{aligned}
R_{0}= & 2 r^{2} a_{2,2} \\
R_{1}= & 4 r a_{1,2}-8 r^{3} a_{3,3} \\
R_{2}= & 24 r^{4} a_{4,4}-20 r^{2} a_{2,3} \\
R_{3}= & -\frac{r^{2}-1}{16} R_{0} \\
& -\frac{r^{2}}{4} \int_{k} \frac{1}{g^{4}(k, 0)} \sum_{\mu}\left\{\sin ^{2} k_{\mu}\left[g(k, 0)-2 r^{2} w^{2}(k)-2 w(k) \cos \left(k_{\mu}\right)\right]^{2}\right\}
\end{aligned}
$$

$R_{0}, R_{1}, R_{2}$ can be calculated from the $a_{n, m}$ 's. $R_{3}$ has to be calculated separately and is given in Appendix A. Notice that $R_{0}, R_{1}, R_{2}$ and $R_{3}$ go to zero for vanishing $r$.

It is immediately apparent that the $R_{0}$ and $m_{q} R_{1}$ terms do not scale appropriately. As a result

$$
m_{\sigma}^{2} \sim R_{0}
$$

and therefore $M_{\sigma}$ is of order cutoff. For the NJL model at large $N$ we can trace the reason for this phenomenon and offer an exact answer. Such a phenomenon may also be responsible for the difficulty in observing a $\sigma$ particle in numerical simulations of QCD with dynamical Wilson fermions.

First we realize that although the Wilson term has raised the doubler masses to the cutoff, the doublers have not disappeared and they can possibly contribute through vacuum polarization effects. We must then try to separate their contribution. Towards this end consider the defining equation of the $\sigma$ propagator eq. 14. The $\tilde{\beta}_{1} / 2$ term contributes to the $\frac{M_{\pi}^{2}}{F_{\pi}^{2}} m_{q}^{2}$ term of eq. 49. This term scales "correctly" and it will not concern us. We focus on $I_{\sigma}$ of eq. 15. $I_{\sigma}(q) \sim \operatorname{Re} \operatorname{Tr} \int_{k} \tilde{M}_{s}^{-1}\left(k-\frac{q}{2}\right) \tilde{M}_{s}^{-1}\left(k+\frac{q}{2}\right)$ where $\left.\tilde{M}_{s}^{(} p\right)$ is the quark propagator at the saddle point and is given in eq. 9. This integral corresponds to a bubble integral with two external legs $\sigma(q)$ and $\sigma(-q)$ and two quarks flowing in the bubble with momenta $k-q / 2$ and $k+q / 2$. Now, each species has momentum that belongs in a section $B_{i}, i=1,2, \ldots 16$ of the Brillouin zone $B$ with extent $\pi / 2$ and $-\pi / 2$ from the origin in each direction (see the first two columns of Table 1). For small $q$ both quarks will have momentum around $k$ and we can then separate the contributions of each species by splitting the integral into the 16 regions $B_{i}$. This way we can isolate the contribution to each term in $I_{\sigma}$ from the propagating quark and the 15 doublers. We will only discuss the contributions to the $R_{0}$ term since $m_{\sigma}^{2} \sim R_{0}$, but similar arguments hold for the $R_{1}, R_{2}$ and $R_{3}$ terms. 
From the definitions in 50, 38, 37 and splitting the integral into 16 regions as described in the previous paragraph we obtain:

$$
R_{0}=2 r^{2} \sum_{i=1}^{16} \int_{B_{i}} \frac{w(k)^{2}}{g_{r}(k, 0)^{2}}
$$

where we have explicitly denoted the $r$ dependence of $g(k, 0)$. It is immediately apparent that the contribution, $R_{0}^{B_{1}}$, form the $B_{1}$ region where the propagating quark lives is not zero. Therefore we can not attribute the whole $R_{0}$ term to the doublers.

The contribution of the propagating quark comes from the high momentum section of the $B_{1}$ region. To see this consider a spherical section $\mathcal{M} \sim m_{q}$ centered around the origin $(0,0,0,0)$ of $B_{1}$. We have:

$$
R_{0}^{B_{1}}=2 r^{2} \int_{\mathcal{M}} \frac{w(k)^{2}}{g_{r}(k, 0)^{2}}+2 r^{2} \int_{B_{1} \cap \mathcal{M}} \frac{w(k)^{2}}{g_{r}(k, 0)^{2}}
$$

Since $W(k) \sim k^{2}$ and $g_{r}(k, 0) \sim k^{2}$ (see eq. 12) we find $\int_{\mathcal{M}} \frac{w(k)^{2}}{g_{r}(k, 0)^{2}} \sim m_{q}^{4}$ and therefore

$$
R_{0}^{B_{1}}=2 r^{2} \int_{B_{1} \cap \mathcal{M}} \frac{w(k)^{2}}{g_{r}(k, 0)^{2}}+O\left(m_{q}^{4}\right)
$$

The Wilson term has not only raised the masses of the doublers but has also changed the high frequency behavior of the propagating quark. In a bubble integral this change is visible.

We could contrive a Wilson term that will raise the doubler masses but not change the high frequency behavior of the propagating quark. This can be done by introducing a momentum dependent $r$ such that:

$$
\begin{aligned}
& r(p)=0 \text { if } p \in B_{1} \\
& r(p)=r \text { if } p \in B_{2}, B_{3}, \ldots B_{16} .
\end{aligned}
$$

Then

$$
R_{0}=2 \int_{B} r(k)^{2} \frac{w(k)^{2}}{g_{r}(k, 0)^{2}}=2 r^{2} \sum_{i=2}^{16} \int_{B_{i}} \frac{w(k)^{2}}{g_{r}(k, 0)^{2}}
$$

and the $\sigma$ mass $m_{\sigma} \sim R_{0}$ will be composed entirely from contributions due to the doublers.

The above discussion can become more transparent for $r \ll 1$. The masses of the 16 species are the roots of $\sum_{\nu} \sin ^{2} k_{\nu}+r^{2}\left(4-\sum_{\nu} \cos k_{\nu}\right)^{2}$. For small $r$ they are given in Table 1.

We calculate the leading order term of the asymptotic expansion of $R_{0}$ in $m_{i}$ and find:

$$
R_{0}=2 r^{2} \int_{B_{1}} \frac{w(k)^{2}}{g_{r=0}(k, 0)^{2}}-2 \sum_{i=1}^{16} m_{i}^{2}\left[r_{1}+s_{1}+s_{1} \log m_{i}^{2}\right]+O(4)
$$

with $r_{1}$ and $s_{1}$ given in eq. 39. The first term comes because of the change of the high frequency behavior of the propagator of each species. The second term clearly shows that $m_{\sigma}^{2}$ can be written as a sum over the doubler masses squared. 


\begin{tabular}{||c|c|c||}
\hline "Brillouin zone" & origin & species mass $+O\left(r^{2}\right)$ \\
\hline \hline$B_{1}$ & 0000 & $m_{1}=0$ \\
\hline$B_{2}$ & $\pi 000$ & $m_{2}=2 r$ \\
\hline$B_{3}$ & $0 \pi 00$ & $m_{3}=2 r$ \\
\hline$B_{4}$ & $00 \pi 0$ & $m_{4}=2 r$ \\
\hline$B_{5}$ & $000 \pi$ & $m_{5}=2 r$ \\
\hline$B_{6}$ & $\pi \pi 00$ & $m_{6}=4 r$ \\
\hline$B_{7}$ & $\pi 0 \pi 0$ & $m_{7}=4 r$ \\
\hline$B_{8}$ & $\pi 00 \pi$ & $m_{8}=4 r$ \\
\hline$B_{9}$ & $0 \pi \pi 0$ & $m_{9}=4 r$ \\
\hline$B_{10}$ & $0 \pi 0 \pi$ & $m_{10}=4 r$ \\
\hline$B_{11}$ & $00 p i \pi$ & $m_{11}=4 r$ \\
\hline$B_{12}$ & $0 \pi \pi \pi$ & $m_{12}=6 r$ \\
\hline$B_{13}$ & $\pi 0 \pi \pi$ & $m_{13}=6 r$ \\
\hline$B_{14}$ & $\pi \pi 0 \pi$ & $m_{14}=6 r$ \\
\hline$B_{15}$ & $\pi \pi \pi 0$ & $m_{15}=6 r$ \\
\hline$B_{16}$ & $\pi \pi \pi \pi$ & $m_{16}=8 r$ \\
\hline & & \\
\hline
\end{tabular}

\section{$4 \quad$ Numerical and large $N$ work on finite lattices}

We simulate the action in eq. 7 for $N=2$ using a standard Hybrid Monte Carlo (HMC) algorithm [14]. We use the conjugate gradient (CG) algorithm without preconditioning to invert the matrix $M^{\dagger} M$ and the leap-frog algorithm to integrate the equations of motion. We measure expectation values of operators that involve the $\sigma$ and $\boldsymbol{\pi}$ fields as well as operators that involve the pseudofermionic fields or traces of appropriate combinations of the matrix $M$.

The reader will realize that the matrix $M$ of eq. 5 of the NJL model does not provide any significant advantages as far as inversion time is concerned over the matrix $M$ of full QCD. The CG will be as time consuming as in full QCD. The advantage comes because of another reason. Since the cutoff of the NJL model is at $\lesssim 1550 \mathrm{MeV}$ and since we will want to look at energies around the pion mass we are dealing with a ratio of scales $\approx \Lambda / M_{\pi} \approx 10$. Therefore we should expect that lattices of size $16^{4}$ should be large enough for this purpose. These size lattices can be simulated in $1 / 4$ of the $64 \mathrm{~K}$ processor CM- 2 supercomputer at SCRI in a reasonable amount of time. Although this was the original justification for performing the numerical simulation it turned out that the numerical results had a more important consequence. As we will demonstrate in Section 4.1 the numerical results on a given finite size lattice are in good agreement with the leading order large $\mathrm{N}$ results on the same size lattice, indicating that the $1 / N$ corrections are small for the quantities we were able to measure.

The large $N$ results on finite size lattices are obtained by explicitly performing the four-dimensional finite momentum sums on a workstation. 
Some of the typical parameters of our numerical simulation are:

* Trajectory length $\tau=1$

* Step size $d \tau \sim 0.02-0.05$

* CG residue $10^{-7}$

* CG iterations $20-160$

$*$ Acceptance rate $>90 \%$

* Mesurements per "point" 100

* Autocorelations $2-10$

* Time for one CG iteration for a $16^{4}$ lattice on $16 \mathrm{~K}$ processors of the SCRI CM-2 $\sim 1.3 \mathrm{sec}$

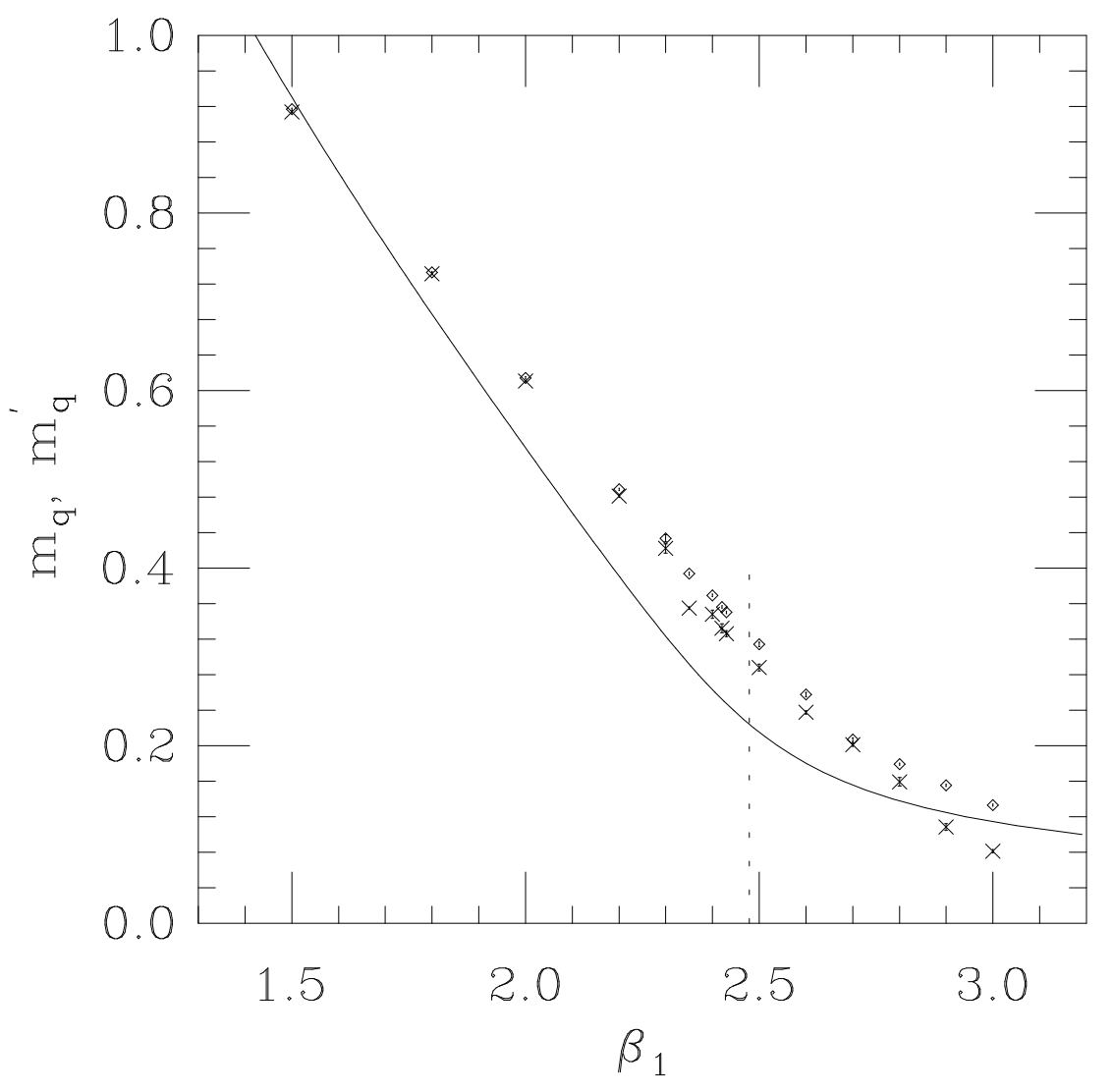

Figure 3. $r=0, m_{0}=0, N=2, L_{x}=8, L_{t}=16$. The diamonds are the values of $m_{q}$ and the crosses are the values of $m_{q}^{\prime}=\left\{-N<\bar{\Psi} \Psi>/\left(2 \beta_{1}\right)\right\}+m_{0}$ from the numerical simulation. Because of the functional identity, eq. 2, the two quantities are expected to be equal. The solid line is the large $N$ prediction on the same size lattice. The dotted line denotes the infinite volume $\beta_{1_{c}}$ from the large $N$ calculation. For $\beta_{1} \leq \beta_{1_{c}}$ the model is in the broken phase. 


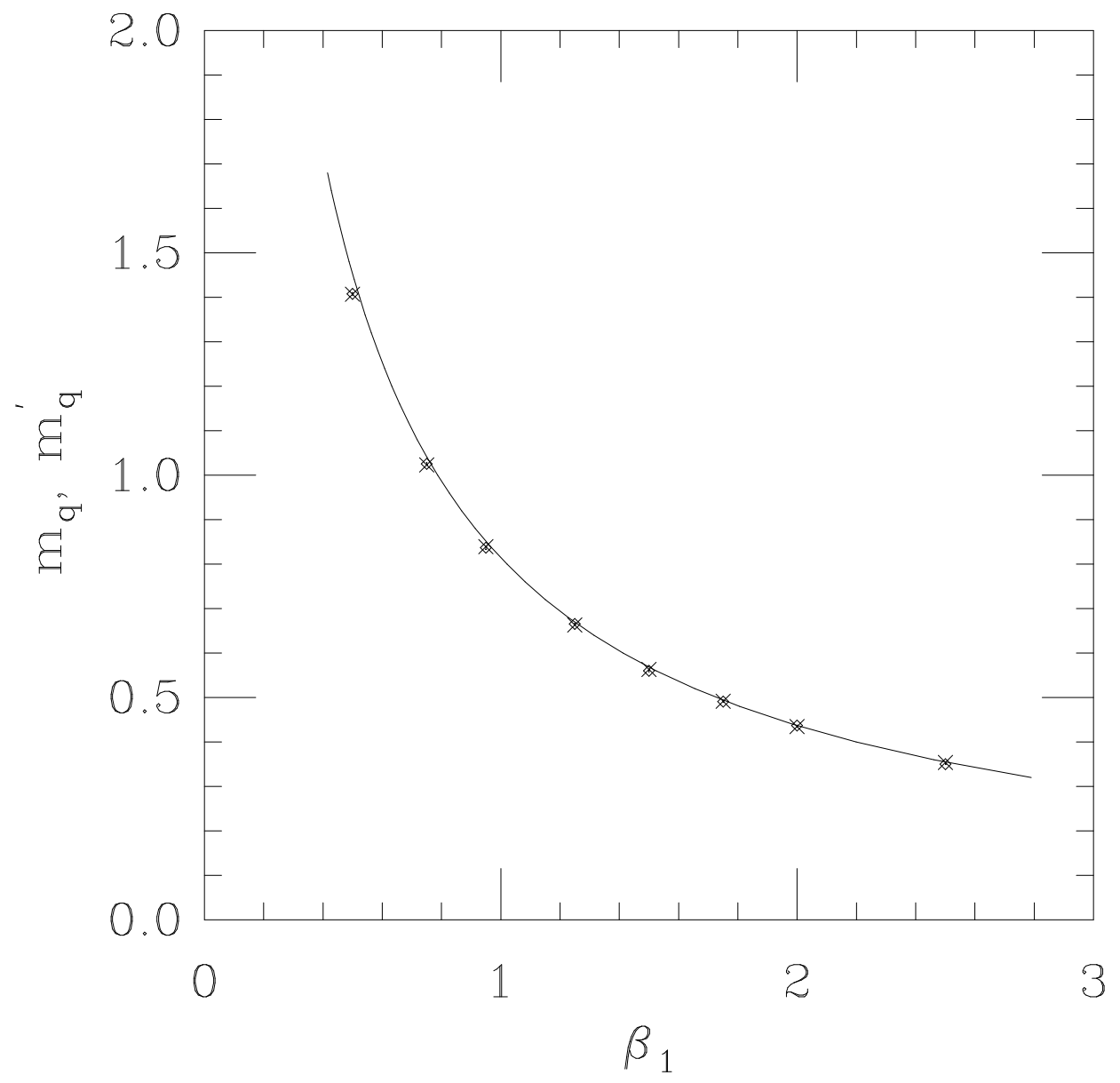

Figure 4. Same as in Figure 3 but for the $U(2) \times U(2)$ NJL and for $r=1, L_{t}=8$. Notice that the diamonds and crosses are almost identical.

\subsection{Numerical results and large $N$}

In this section we present our numerical results for $N=2$ and we compare them with the large $N$ predictions (scaled to $N=2$ when necessary) on same size lattices.

To check for consistency of the fermionic and auxiliary fields we plot $m_{q}$ and $m_{q}^{\prime}=$ $\left\{-N<\bar{\Psi} \Psi>/\left(2 \beta_{1}\right)\right\}+m_{0}$ vs. $\beta_{1}$ as determined from the numerical simulation. Because of the functional identity, eq. 2, we should have $m_{q}=m_{q}^{\prime}$. This relation is satisfied nicely.

The agreement with large $N$ of dynamically determined quantities vs. bare quantities is quite good and helps us to get oriented in the bare parameter space. This can be seen for various values of the parameters in Figures 3, 4, 5, and $7 \mathrm{a}$. 
The important comparison with large $N$ that will help us get a feel for the size of the $1 / N$ corrections comes from comparisons of dynamically determined quantities vs. other dynamically determined quantities. In particular we exchange one of the bare parameters for $\langle\sigma\rangle$.

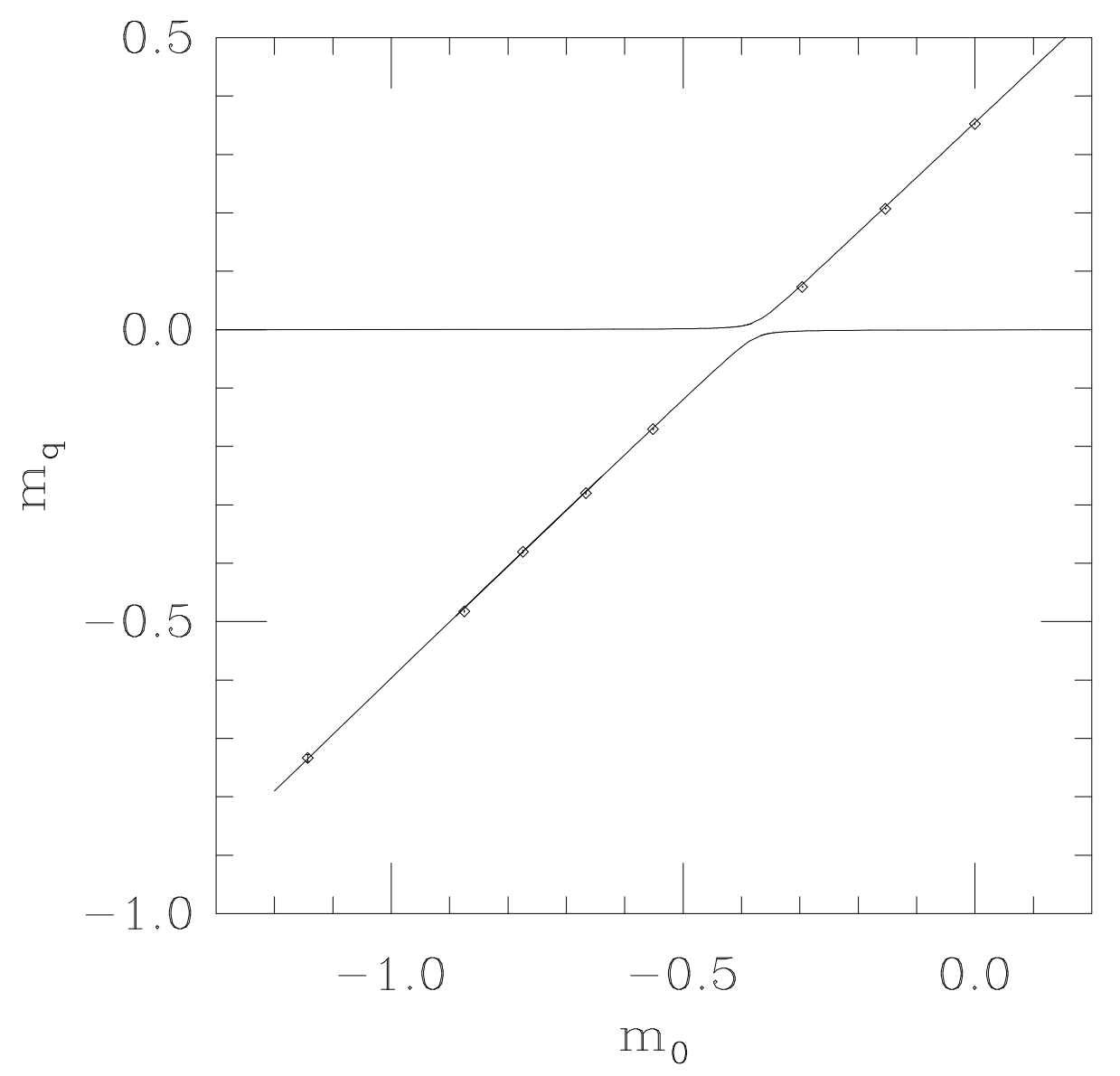

Figure 5. $r=1, \beta_{1}=2.5, N=2$. The diamonds are the values of $m_{q}$ from the numerical simulation for $L_{x}=8, L_{t}=16$. The solid line is the large $N$ result on the same size lattice.

In Figure 6 we present the $\sigma$ and $\boldsymbol{\pi}$ propagators in momentum space for ten small momenta and $r=0$. It is from this figure that we would have to extract $Z_{\pi}$. As it can be seen the large $N$ prediction for the same $\langle\sigma\rangle$ as the one measured in the simulation is in good agreement with the numerical results. The large $N$ predictions in Figures 6a, 6b, $6 \mathrm{c}$ and $6 \mathrm{~d}$ "fit" the numerical results with $\chi^{2}$ per degree of freedom $0.38,0.67,0.32,0.42$ respectively. This means that the determination of $f_{\pi}=\langle\sigma\rangle \sqrt{\frac{N}{Z_{\pi}}}$ as a function of $\langle\sigma\rangle$ has small $1 / N$ corrections. 

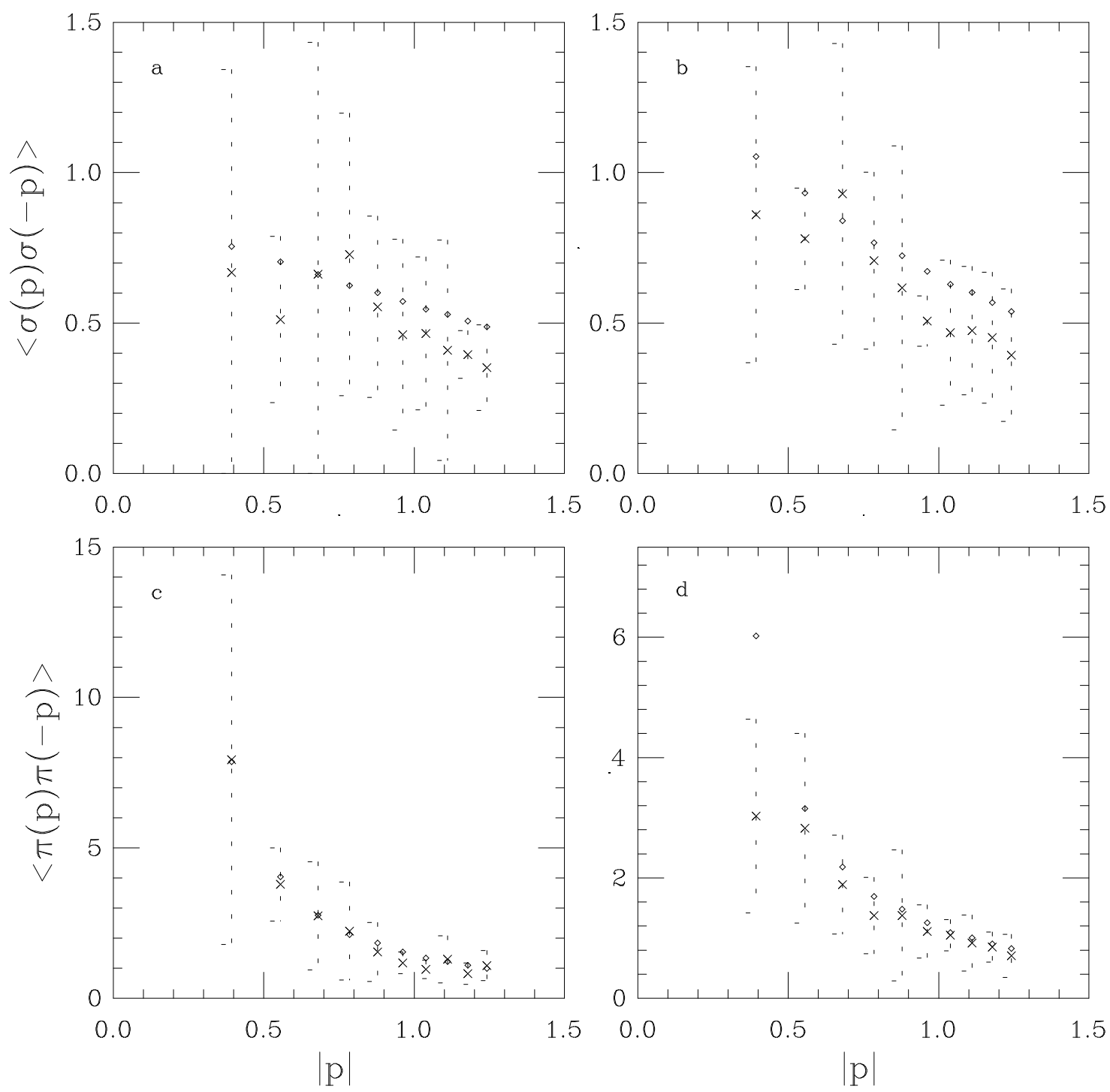

Figure 6. a) The $\sigma$ propagator in momentum space for 10 small momenta with $r=0$, $m_{0}=0, \beta_{1_{M C}}=2.2,\langle\sigma\rangle=\sigma_{s}=0.4840, N=2, L_{x}=16, L_{t}=16$. The crosses are the values from the numerical simulation. The diamonds are the large $N$ results and "fit" the numerical results with a $\chi^{2} /$ d.o.f. $=0.38$. b) Same as in 6 a but for $\beta_{1_{M C}}=2.4$, $\langle\sigma\rangle=\sigma_{s}=0.35$. The large $N$ results "fit" the numerical results with a $\chi^{2} /$ d.o.f. $=0.67$. c) Same as in Figure 6a but for the pion propagator. The large $N$ results "fit" the numerical results with a $\chi^{2} /$ d.o.f. $=0.32$. d) Same as in $6 \mathrm{~b}$ but for the pion propagator. The large $N$ results "fit" the numerical results with a $\chi^{2} /$ d.o.f. $=0.42$.

Another dynamically determined quantity that agrees well with the large $N$ prediction when plotted vs. $\langle\sigma\rangle$ is the pion mass $m_{\pi}$. This plot is shown in Figure $7 \mathrm{~b}$. This figure suggests that the $1 / N$ corrections to $m_{\pi}$ are fairly small. In this figure, as well as in figure $7 \mathrm{a}, m_{\pi}$ was not calculated from the definition 16 but as the imaginary pole of $G_{\pi}$ (see eq. 114). 


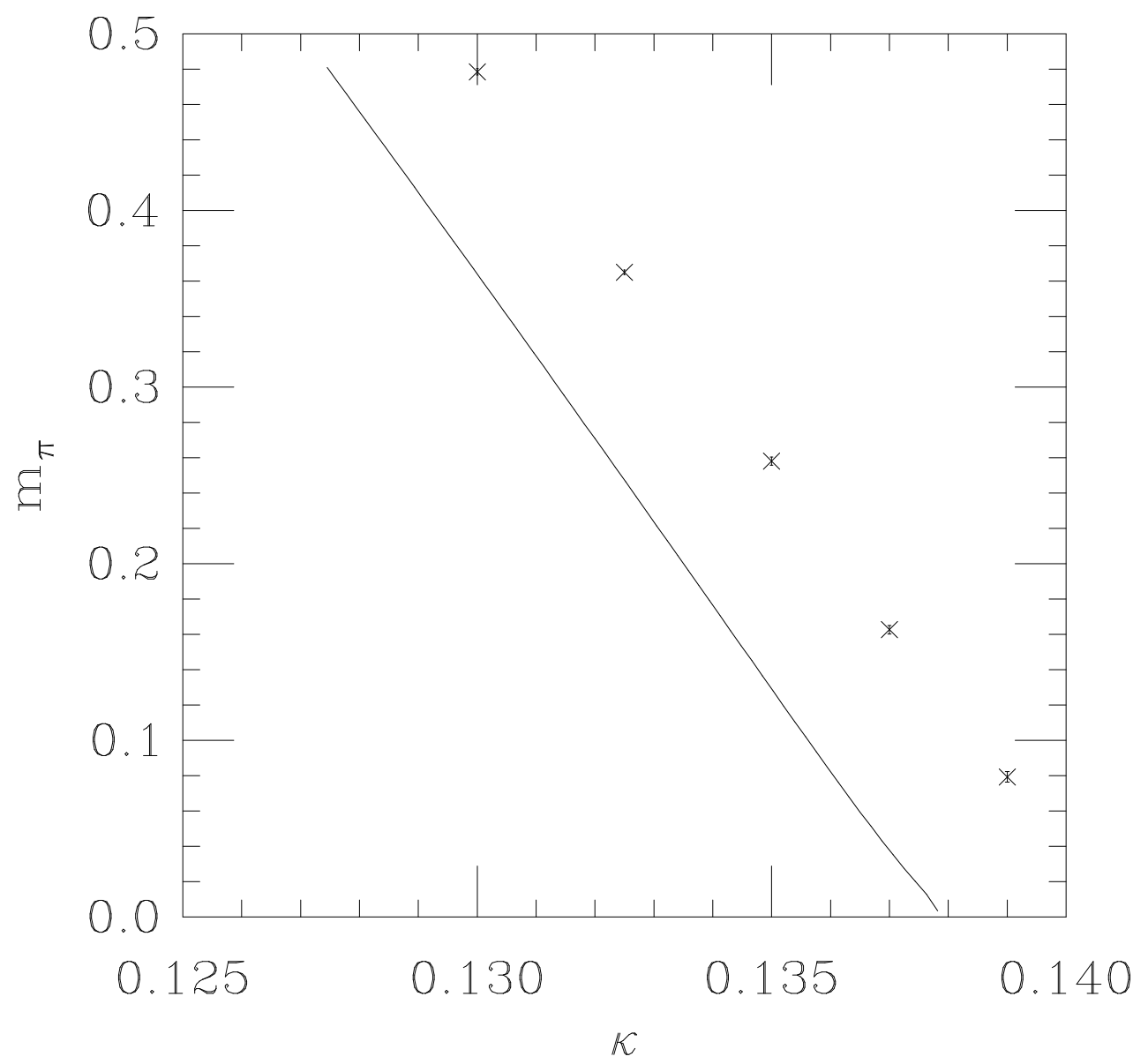

Figure 7a. $\beta_{1}=2.5, r=1, N=2, L_{x}=8, L_{t}=16$. The crosses are the MC data. The solid line is the large $N$ prediction on the same size lattice.

It should be noted that the good agreement of the large $N$ results with the numerical simulations is not only present for naive fermions where the number of species is 16 times larger and therefore one would have naively expected the leading order large $N$ expansion to be a good approximation. It is also present for Wilson fermions $(r \neq 0)$ as it can be seen from Figures 4, 5, 7a and 7b.

Finally, as discussed in detail in Section 3.2, for $r \neq 0$ the $\sigma$ mass is of order cutoff and therefore very heavy to be able to measure from the decay of the $\sigma-\sigma$ correlation function. However, for $r=0$ one would expect to be able to measure $m_{\sigma}$. As we will discuss in the next section this is not possible with the lattice sizes accessible to us. This is unfortunate since $m_{\sigma}$ is another very important quantity. However, we expect the size of the $1 / N$ corrections of $m_{\sigma}$ to be similar to the ones of $m_{\pi}$ and therefore fairly small. Also, 
measurements of the sigma width were not performed, but, as discussed in Section 3.1, we expect the $1 / N$ corrections to the width to be fairly large.

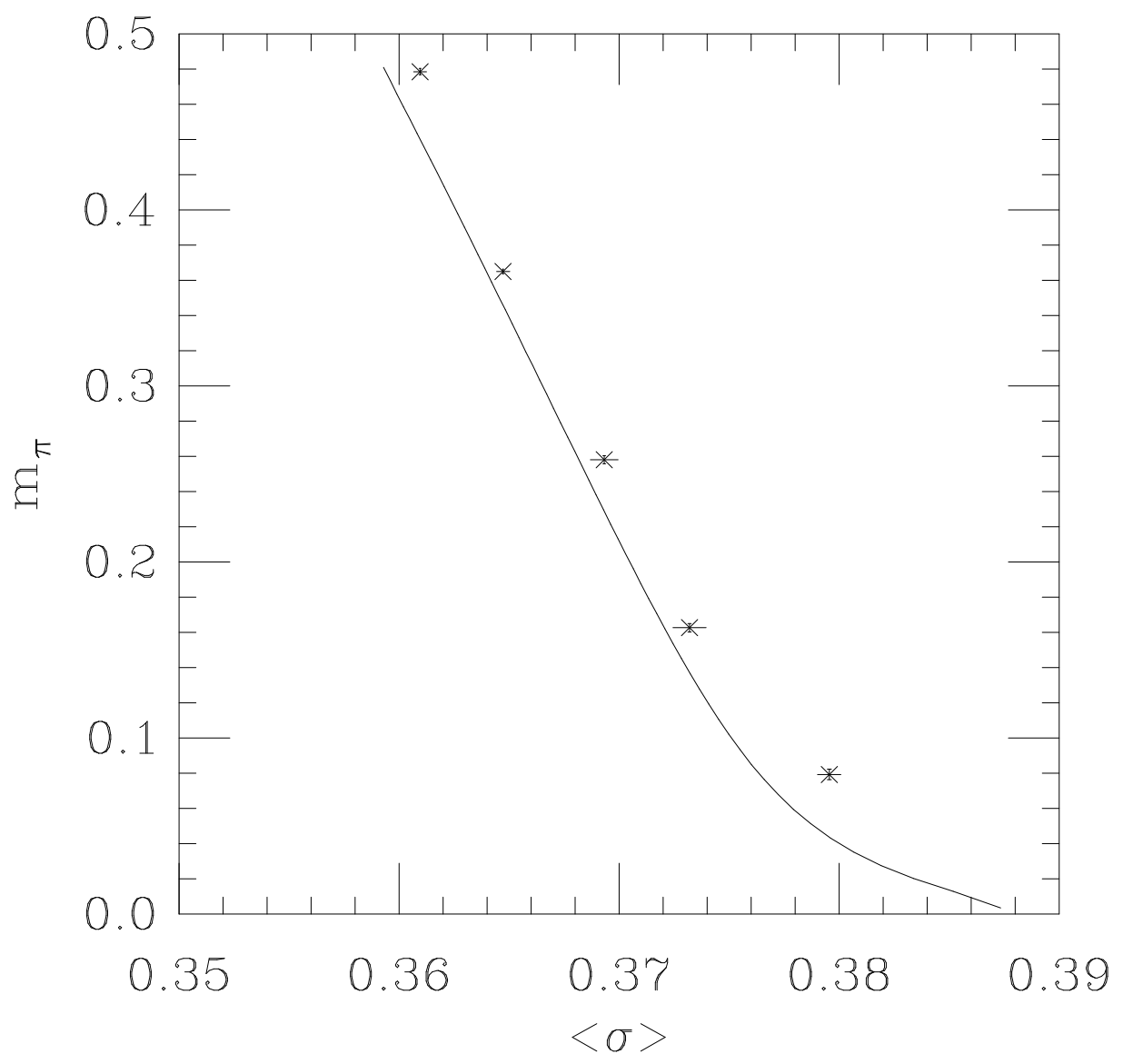

Figure $7 \mathbf{b}$. Same as in Figure 7 a but as a function of $\langle\sigma\rangle$.

\subsection{The zero momentum mode of the quarks}

To leading order at large $N$ the matrix $M^{\dagger} M$ of eq. 5 is diagonal in momentum, spin, flavor, and color spaces.

$$
M^{\dagger} M=M_{s}^{\dagger} M_{s}=\sum_{\mu} \sin ^{2} p_{\mu}+\left[m_{q}+r\left(4-\sum_{\nu} \cos p_{\mu}\right)\right]^{2}+O(1 / N)
$$

The smallest eigenvalue of this matrix is $m_{q}^{2}$ and corresponds to the $p=0$ matrix element. This in turn corresponds to the zero momentum modes of the quarks which from now on we will simply refer to as "zero modes". 


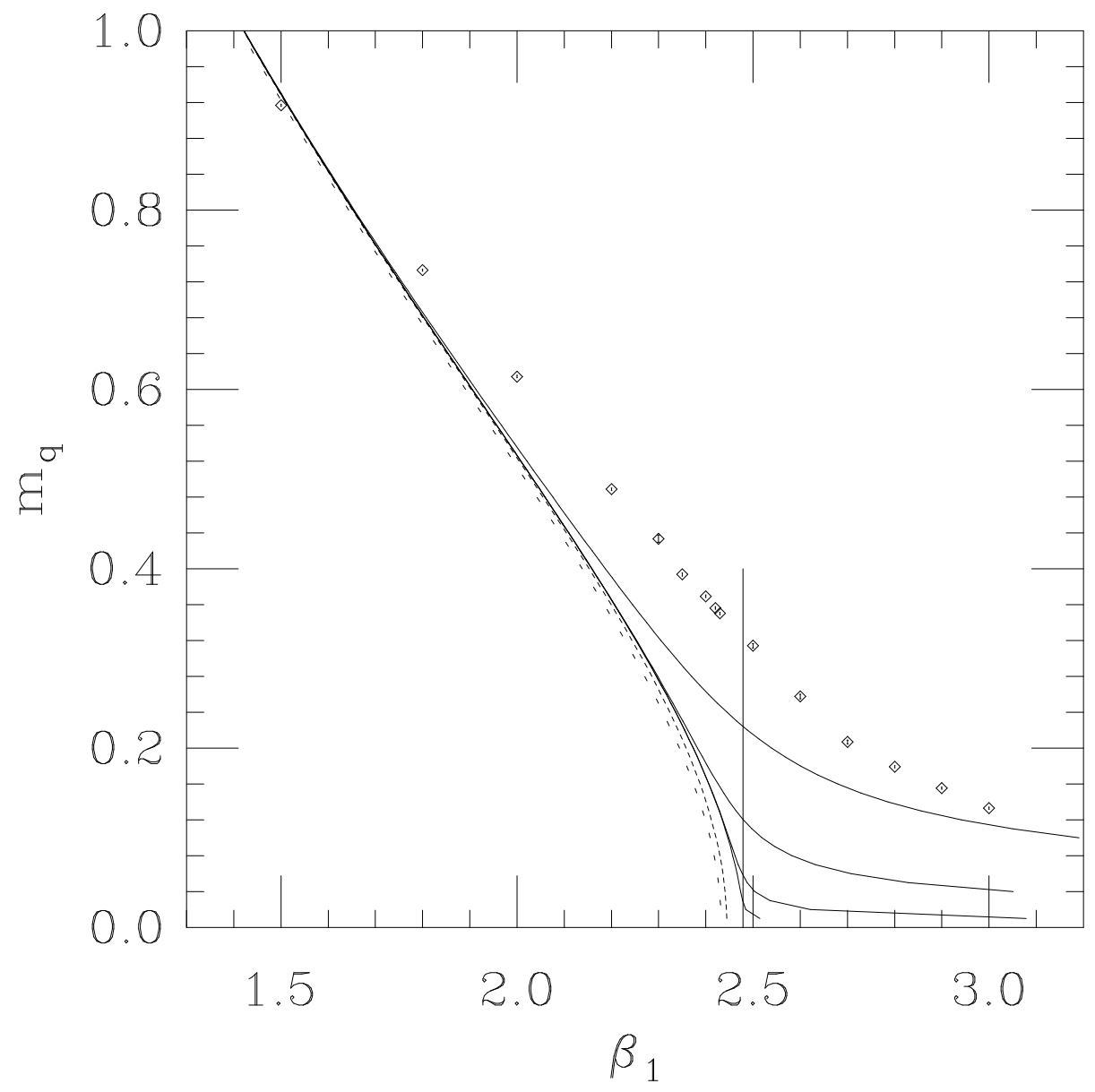

Figure 8. $r=0, m_{0}=0, N=2$. The diamonds are the values of $m_{q}$ from the numerical simulation for $l x=8, l t=16$. The solid lines are the large $N$ numbers with the zero mode included. From right to left they correspond to $\left(L_{x}=8, L_{t}=16\right),\left(L_{x}=16, L_{t}=16\right)$, $\left(L_{x}=32, L_{t}=32\right),\left(L_{x}=64, L_{t}=64\right)$. The dotted lines are the large $N$ numbers with the zero mode excluded. From right to left they correspond to $\left(L_{x}=16, L_{t}=16\right)$, $\left(L_{x}=8, L_{t}=16\right)$. The solid vertical line denotes the infinite volume $\beta_{1_{c}}$ from the large $N$ calculation. For $\beta_{1} \leq \beta_{1_{c}}$ the model is in the broken phase.

For small $m_{q}$ the condition number $c$ of this matrix is:

$$
\begin{aligned}
& c \approx \frac{4}{m_{q}^{2}} \text { for } r=0 \\
& c \approx \frac{64 r^{2}}{m_{q}^{2}} \text { for } r=1
\end{aligned}
$$


A large condition number will make the inversion of $M^{\dagger} M$ very slow. An important observation can be made by noticing the dependence of the condition number on $r$. This suggests that performing the simulation with smaller $r$ will yield a quite faster inversion. It is possible that this may also be the case for QCD.

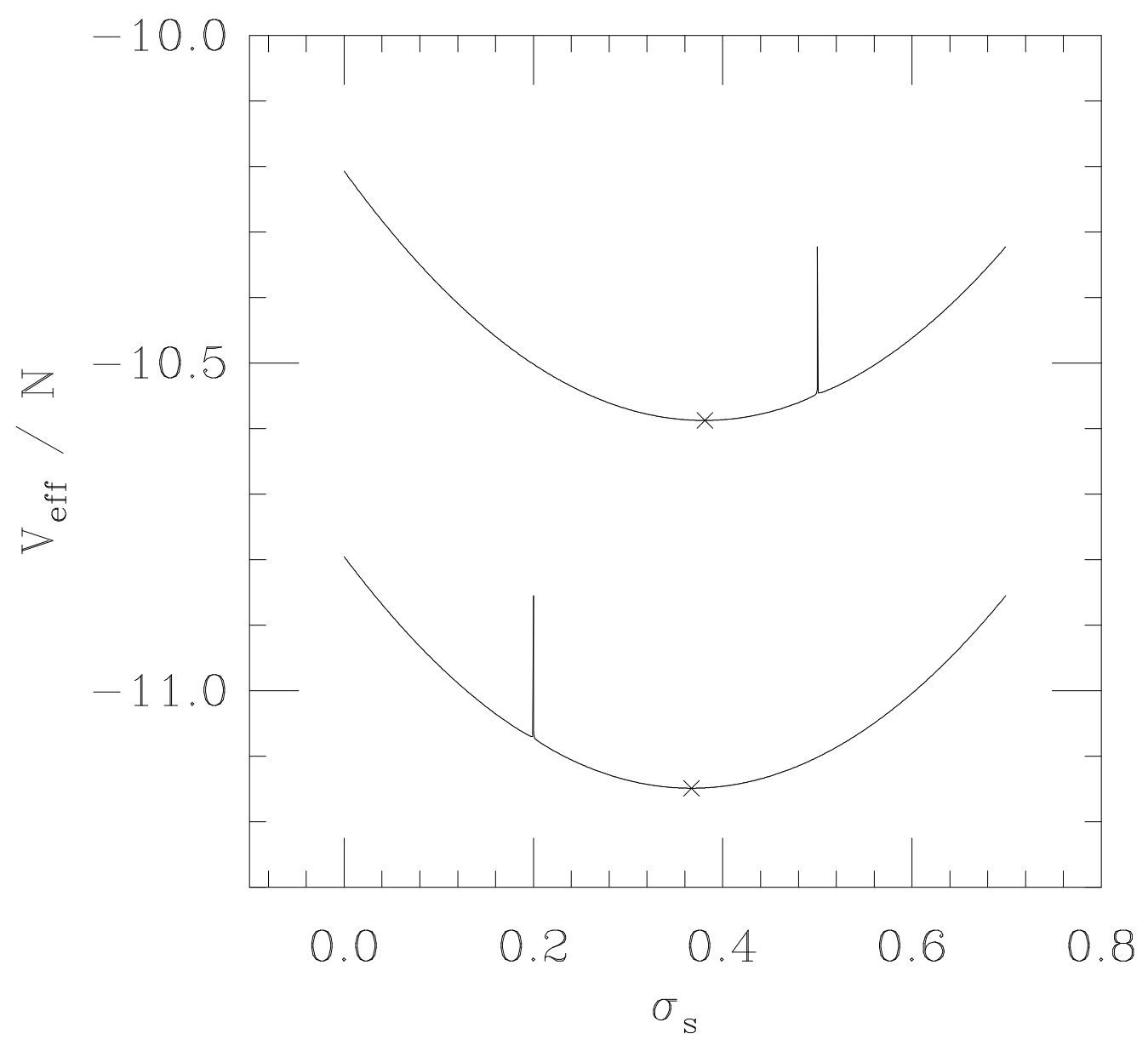

Figure 9. $r=1, \beta_{1}=2.5, N=2, L_{x}=8, L_{t}=16$. The lines are the large $N$ effective potential with $m_{0}=-0.2$ (bottom) and $m_{0}=-0.5$ (top). The crosses indicate the absolute minimum. $V_{e f f}$ is infinite at the spikes.

But the unwelcomed effect of the zero modes on a finite lattice is not limited to large inversion times. Because on a finite lattice their effects are not suppressed by the measure but instead by an inverse volume factor, it turns out that in certain cases they severely obscure the physics. 


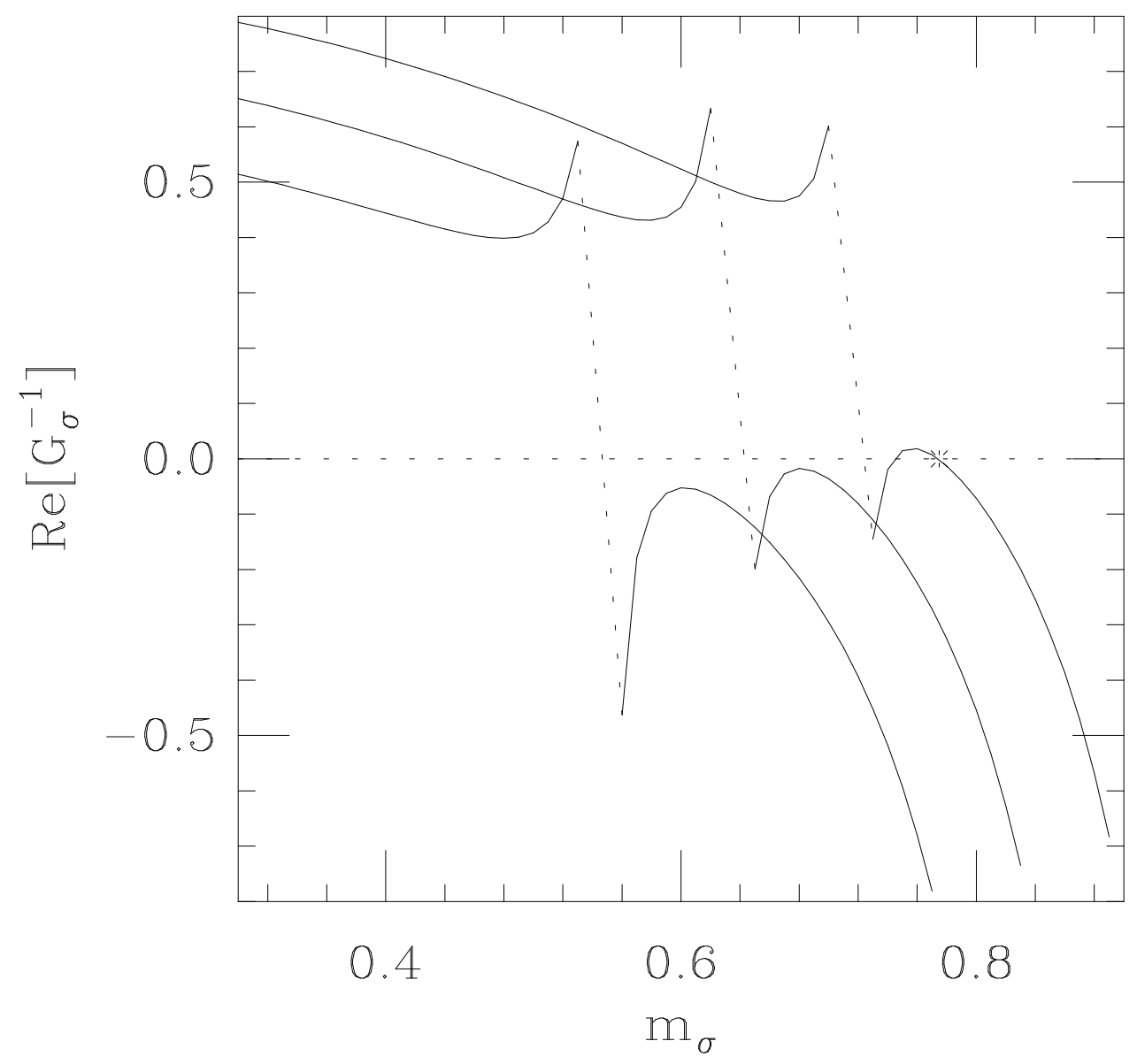

Figure 10a. $r=0, m_{0}=0, N=2, L_{x}=16, L_{t}=16$. The solid lines are the real part of the inverse $\sigma$ propagator for external 4 -momentum $q=\left(i m_{\sigma}, 0,0,0\right)$ at large $N$ with the zero modes included. From left to right they correspond to $\beta_{1}=2.3,2.25,2.2$ or equivalently to $\sigma_{s}=0.278,0.323,0.366$. The functions have discontinuities that are denoted with the doted lines. The star denotes the relevant zero for the $\beta_{1}=2.2$ line.

In Figure 8 we plot $m_{q}=\langle\sigma\rangle$ vs. $\beta_{1}$ for $r=0, m_{0}=0, L_{x}=8$, and $L_{t}=16$ (same as Figure 3 ). By simply looking at the numerical results we would not only be unable to estimate the critical point but also we would be unable to see any indication of a phase transition. The large $N$ result on the same size lattice also has the same problems. As we increase the lattice size in the large $N$ calculation (solid lines from top to bottom) we see that a picture of an order parameter slowly materializes. At $L_{x}=64, L_{t}=64$ a fairly good prediction of the large $N$ infinite volume critical point is achieved. If we now do the same large $N$ calculation but neglect from the momentum sums the zero modes, we obtain as a 
result the two dotted lines for $L_{x}=8, L_{t}=16$ and $L_{x}=16, L_{t}=16$ (from left to right). We see that neglecting the zero modes on an $L_{x}=8, L_{t}=16$ lattice gives very similar results as the ones obtained on a $L_{x}=64, L_{t}=64$ lattice with the zero modes included.

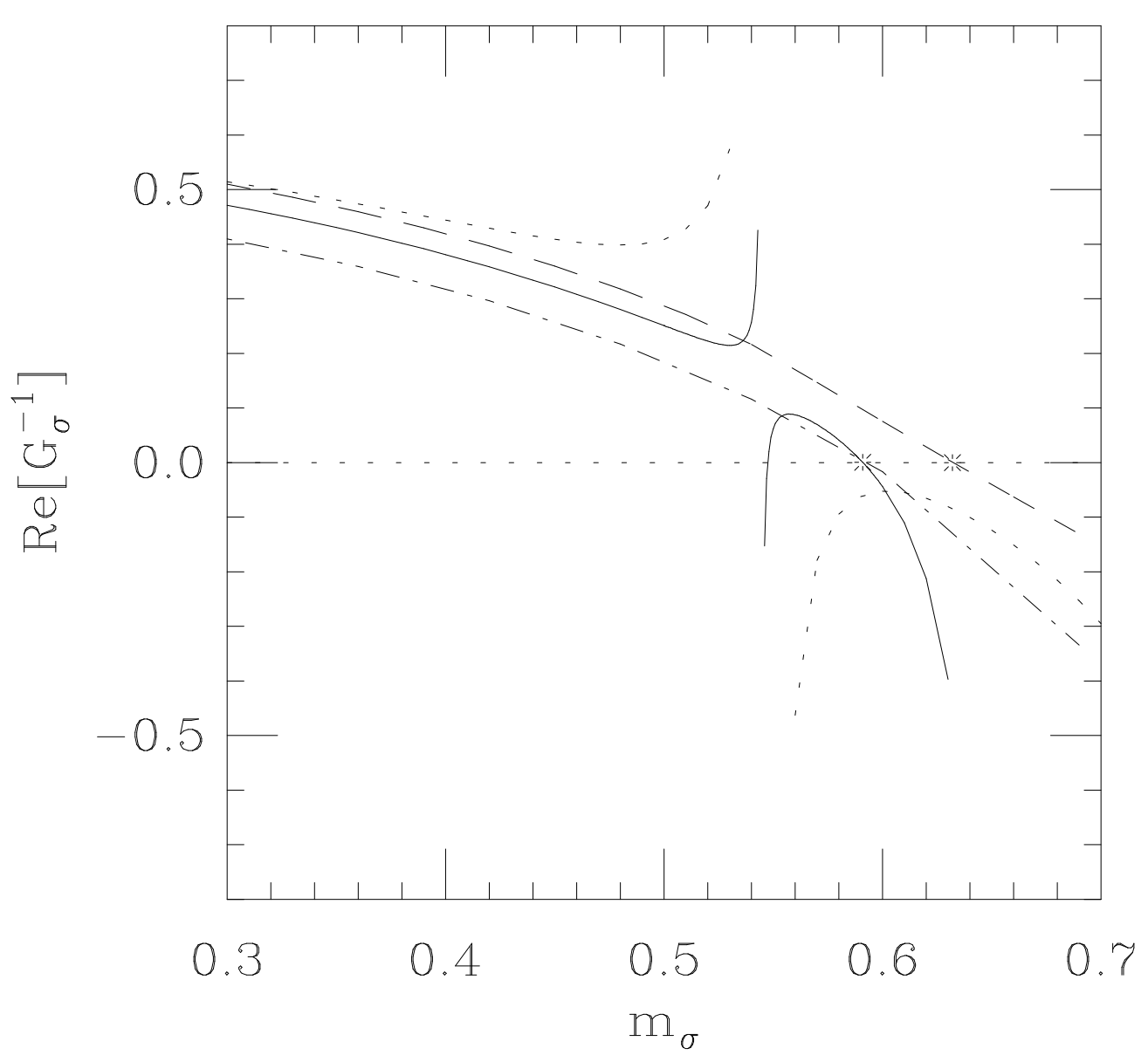

Figure 10b. $r=0, m_{0}=0, N=2, \beta_{1}=2.3$ The lines are the real part of the inverse $\sigma$ propagator for external 4 -momentum $q=\left(i m_{\sigma}, 0,0,0\right)$ at large $N$. The dotted line is for $L_{x}=16, L_{t}=16$ with the zero mode included, the solid line for $L_{x}=32, L_{t}=32$ with the zero mode included, the dot-dash line is for $L_{x}=16, L_{t}=16$ with the zero mode excluded, and the dashed line is the infinite volume result from the asymptotic expansion. The stars denote the relevant zeros.

If we plot the effective potential of eq. 11 vs. $\sigma_{s}$ for a finite lattice we will obtain a result as in Figure 9. The "spike" is a result of the presence of the zero mode on the lattice sum and extends to infinity. The presence of this spike may create thermalization problems if the initial configuration is chosen on the "wrong" side of the "spike". Of course 
the "width" of the spike is negligible and therefore this problem may not be important.

As mentioned at the end of the previous section, although one would expect to be able to measure the $\sigma$ mass in the $r=0$ case we were not able to do so. Large $N$ provides an explanation of this unexpected problem. In Figure 10a we plot the real part of the inverse $\sigma$ propagator, eq. 14, for a finite lattice with $L_{x}=16, L_{t}=16$ and external momentum set to $q=\left\{\mathrm{im}_{\sigma}, 0,0,0\right\}$. The sigma mass should be obtained at the zero of this function. We see that because of the presence of a discontinuity we do not obtain a root until $m_{\sigma}$ becomes heavy. The presence of this discontinuity is again due to the zero modes.

In Figure 10b we plot the left most case of Figure 10a (dotted line). As we already mentioned there is no zero. If we increase the lattice size to $L_{x}=32, L_{t}=32$ (solid line) we see that a zero develops. The infinite volume result from the asymptotic expansion (dashed line) has a zero nearby and no discontinuity since the zero modes are fully suppressed. If on the $L_{x}=16, L_{t}=16$ lattice we now exclude the zero modes (dot-dash line) we see that the discontinuity disappears and a zero very close to the infinite volume result is obtained.

\subsection{The $m_{\pi}=0$ line on a finite lattice at large $N$}

The $m_{\pi}=0$ zero line is of particular importance since it is there that the continuum chiral limit is obtained. As discussed in Section 3.2 this limit is obtained in the part of the $m_{\pi}=0$ line that corresponds to small quark mass. That region was presented in Figures $2 \mathrm{a}, 2 \mathrm{~b}$ for $-0.2 \leq m_{q} \leq 0.3$. If, for the same values of $m_{q}$, the zero pion mass line is calculated on a finite volume one finds that the corresponding ranges of $\kappa$ and $\beta_{1}$ change by almost an order of magnitude. To be more specific, for $r=1$ the point of the the zero pion mass line corresponding, for example, to $m_{q}=0.015$ has $m_{0}=-2.75$ and $\beta_{1}=0.34$ at infinite volume (see Figure 2a). The same point on an $L_{x}=8, L_{t}=16$ lattice has $m_{0}=-0.37$ and $\beta_{1}=2.51$. This change is fairly unusual and it may appear as if there is a contradiction between the finite and infinite volume results. In particular, in the infinite volume work of [10 no point with $m_{\pi}=0$ was found for $\beta_{1}>1.41$. To clarify this issue we plot in Figure 11 a the $m_{\pi}=0$ line for an $L_{x}=8, L_{t}=16$ lattice for $-\infty<m_{q}<+\infty$. This line is calculated as follows: For a given value of $m_{q}$ we use the finite volume version of eq. 46

$$
m_{0} \frac{1}{L_{x}^{3} L_{t}} \sum_{k} \frac{1}{g\left(k, m_{q}\right)}+r \frac{1}{L_{x}^{3} L_{t}} \sum_{k} \frac{w(k)}{g\left(k, m_{q}\right)}=0
$$

to calculate $m_{0}$. Next, using this $m_{q}$ and $m_{0}$ we calculate $\beta_{1}$ by combining equations 10 and 13. The finite volume version of this is:

$$
\beta_{1}=\frac{2 N}{m_{q}-m_{0}} \frac{1}{L_{x}^{3} L_{t}} \sum_{p} \frac{m_{q}+r w(p)}{g\left(p, m_{q}\right)} .
$$

At point "1" of Figure 11a $m_{q}$ is very large and positive. As $m_{q}$ decreases, crosses zero and tends to very large negative values, we transverse the whole solid line from "1" to "2" to " 3 " ... to " 12 " where $m_{q}$ is very large and negative. The "prongs" of this figure, points "3", "5", "7", "9", and " $11 "$ ", extend all the way to $\beta_{1}=\infty$ where they correspond to

$m_{q}=0,-2,-4,-6$, and -8 , respectively. This singular behavior originates from the terms of the momentum sum in eq. 61 that correspond to the origins of the "Brillouin zones" 
of the 16 species (see Table 1). When these terms are neglected from the calculation (the contribution of these terms disappears in the infinite volume limit) we obtain the dotted line of Figure 11a.

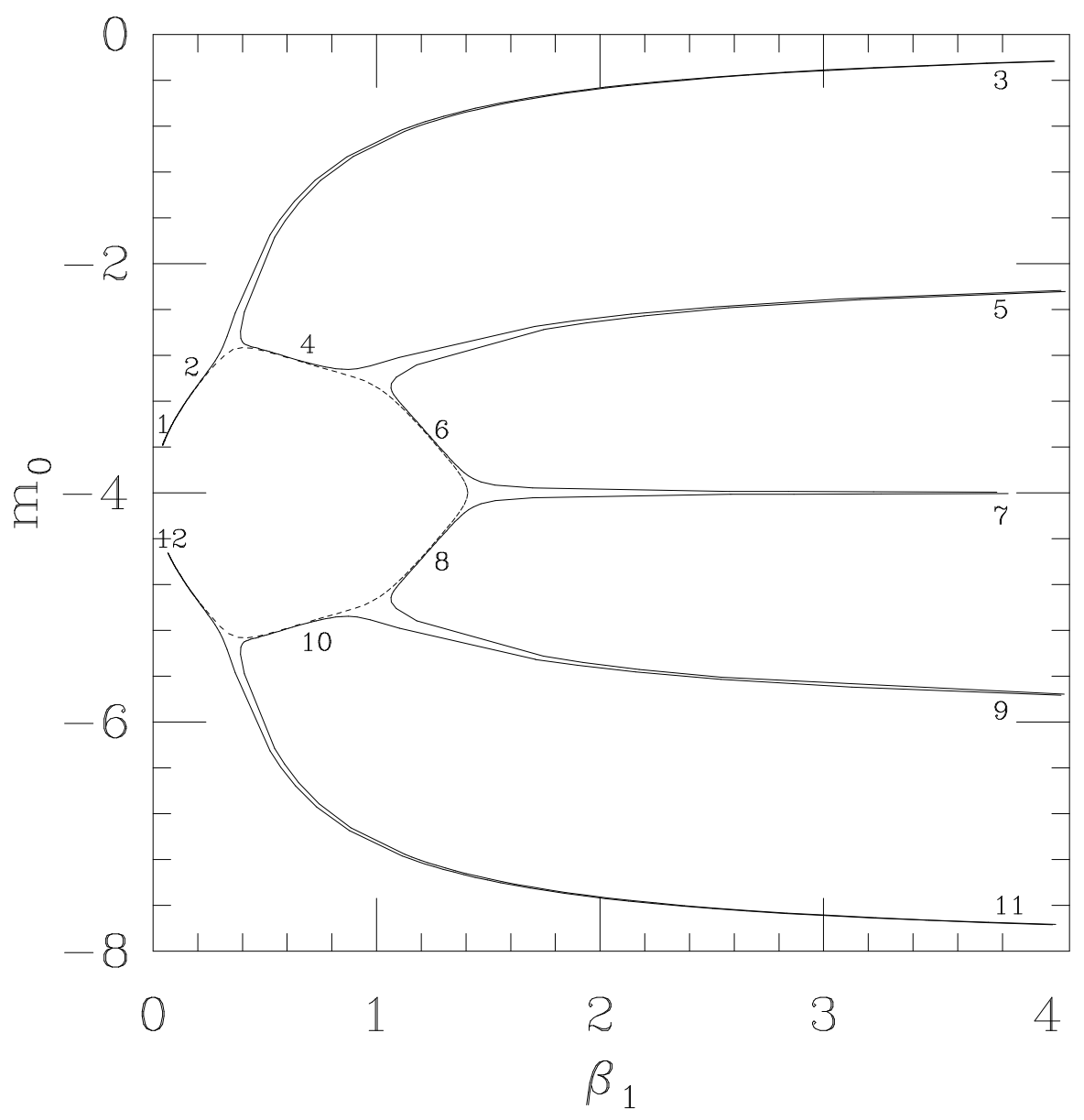

Figure 11a. $r=1, N=2, L_{x}=8, L_{t}=16$. The solid line denotes the $m_{\pi}=0$ line.

To see more clearly how this behavior develops as we increase the volume we concentrate, as an example, on the "prong" that corresponds to $m_{q}=0$ since it is this "prong" that is most interesting in the recovery of continuum physics. A blown up picture of this "prong" is plotted in Figure 11b. The outer solid lines correspond to the $L_{x}=8, L_{t}=16$ lattice. Moving inwards the solid lines correspond to an $L_{x}=16, L_{t}=16$ lattice and an $L_{x}=32$, $L_{t}=32$ lattice. The dashed line corresponds to the $L_{x}=8, L_{t}=16$ lattice but with the singular terms removed. The dotted line is the infinite volume result of Figure $2 \mathrm{a}$ for $-0.1 \leq m_{q} \leq 0.1$. Because the singularities are only quadratic they have disappeared in the infinite volume limit. The circle corresponds to the infinite volume $m_{q}=0, m_{\pi}=0$ 
point. As the volume increases the whole "prong" is slowly mapped down to the infinite volume result of the dotted line. It is also very interesting to notice how close the infinite volume result is to the $L_{x}=8, L_{t}=16$ lattice result with the singular terms removed. Similar behavior is expected for the other "prongs" as well.

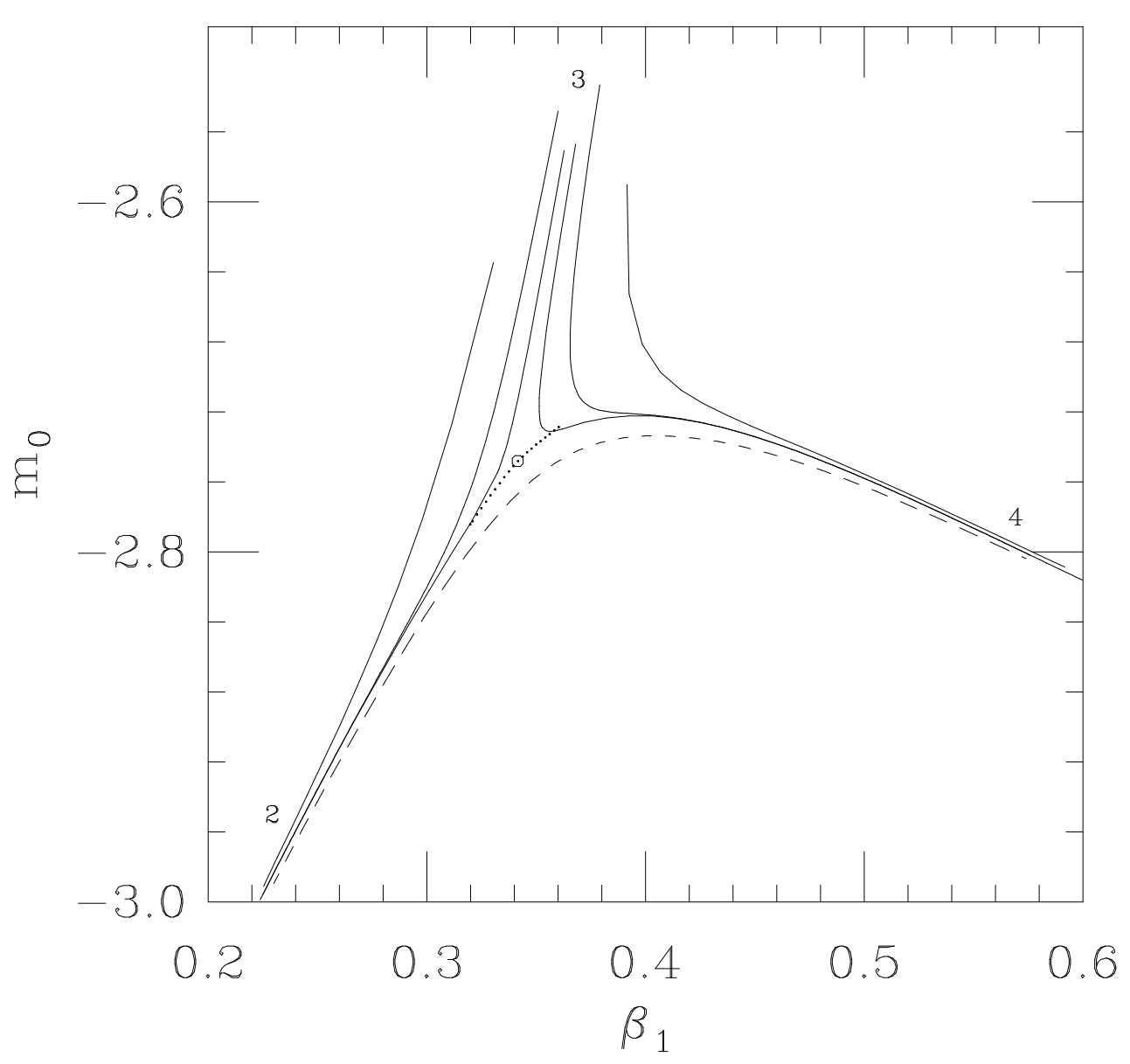

Figure 11b. Detail around the "prong" that corresponds to $m_{q}=0$ ("prong" "3" of figure 11a).

In [10] it was shown that the $m_{\pi}=0$ line is the phase line that separates the parityflavor symmetric phase $(\langle|\boldsymbol{\pi}|\rangle=0)$ with the spontaneously broken parity-flavor symmetry phase $(\langle|\boldsymbol{\pi}|\rangle \neq 0)$. We have confirmed that this is also the case on the finite lattice. The region inside the octopus-like graph of Figure 11a has $(\langle|\boldsymbol{\pi}|\rangle \neq 0)$.

Finally, notice that the numerical results for $m_{\pi}$ of Figure 7 a indicate that on a finite lattice $m_{\pi}$ can be made quite small. The place where this happens is predicted by large $N$ quite well and it corresponds to a point on "prong" " 3 " that is quite far from where the 
corresponding point of the infinite volume would be (somewhere on the dotted line on the "inside" of the "prong").

\section{Conclusion and Summary}

In this paper we have found that the lattice version of the NJL model is an excellent toy model to investigate issues related to lattice QCD. We have used the large $N$ approximation to leading order in $1 / N$ to obtain non perturbative analytical results over almost the whole parameter range. By using numerical simulations of the model we estimated that for most of the quantities we considered the $1 / N$ corrections are small.

The main results of our investigation are listed below:

1) With Wilson fermions we obtain at large $N$ analytical expressions of the pion mass $\left(m_{\pi}\right)$ and quark mass $\left(m_{q}\right)$ in lattice units as functions of the bare parameters of the model. We are then able to make exact statements regarding the approach to the continuum chiral limit. The "phase diagram" is presented in Figure 2. This may provide an insight on how the retrieval of the continuum chiral limit is achieved in QCD.

2) At large $N$ and for Wilson fermions the $\sigma$ particle has mass proportional to the cutoff. Our analysis traces this fact to two related reasons. First, although the Wilson term has raised the masses of the doublers to the cutoff, it has not decoupled them from the theory. Through vacuum polarization these contribute to the $\sigma$ self energy and raise its mass. Second, although the Wilson term has not altered the low frequency behavior of the propagating quark, it has however altered its high frequency behavior. Again through vacuum polarization the high frequency modes contribute to the $\sigma$ self energy and also raise its mass. Such a phenomenon may also be responsible for the difficulty in observing a $\sigma$ particle in numerical simulations of QCD with dynamical Wilson fermions.

3) The numerical simulation is performed on finite lattices. For naive fermions one would expect to be able to see some indication of the chiral phase transition as well as a $\sigma$ particle. However, by simply looking at the graph of the vacuum expectation value vs. the coupling (see fig. 8) on an $8^{3} \times 16$ lattice one can not see any indication of a phase transition. Also the $\sigma$ particle in a $16^{4}$ lattice is either non existent or too heavy to be measured. Both of these unexpected results can be explained at large $N$. The reason is traced to the existence of zero quark momentum modes that on a finite lattice are not sufficiently suppressed. The zero modes besides obscuring some of the physics are also probably partially responsible for the large inversion times in the HMC algorithm. To leading order at large $N$ the smallest

eigenvalue of the matrix that has to be inverted is $m_{q}^{2}$ and corresponds to the zero quark momentum mode. For small $m_{q}$, the condition number of the matrix is $4 / m_{q}^{2}$ for $r=0$ and $64 r^{2} / m_{q}^{2}$ for $r=1$. An important observation can be made by noticing the dependence of the condition number on $r$. This suggests that performing the simulation with smaller $r$ will yield a quite faster inversion. It is possible that this may also be the case for QCD.

4) The observables measured in the numerical simulation (chiral condensate, vacuum expectation value, pion wave function renormalization constant, pion mass) have values that are in good agreement with leading order large $N$. This provides a quantitative prediction for the size of the $1 / N$ corrections. In agreement with the large $N$ predictions discussed 
in 2 and 3 above, the $\sigma$ mass was very heavy to give a good signal and was not measured. Also, measurements of the sigma width were not performed, but, as it will be discussed in 5 below, we expect the $1 / N$ corrections to the width to be large.

5) For naive fermions we calculate at large $N$ and with $M_{\pi}=140 \mathrm{MeV}$ the $\sigma$ mass $\left(M_{\sigma}\right)$, the $\sigma$ width $\left(\Gamma_{\sigma}\right)$ and the quark mass $\left(M_{q}\right)$ in physical units as functions of the cutoff. By setting $M_{q}=310 \mathrm{MeV}$ we find $M_{\sigma}=726 \mathrm{MeV}, \Gamma_{\sigma}=135 \mathrm{MeV}$, and $\Lambda=\pi / a=1150 \mathrm{MeV} . M_{\sigma}$ is consistent with phenomenological expectations and $\Lambda$ is consistent with the expectation that the cutoff should be close and below the mass of the lightest glueball $(1550 \mathrm{MeV})$. The width however is underestimated. The reason is traced to the fact that to leading order in large $N$ the width receives contributions only from the quark bubble and not from the pion bubble because the pion bubble is of order $1 / N$. Because the phase space available for the $\sigma$ to decay to two quarks is much smaller than the phase space to decay to two pions the pion loop contribution although of order $1 / N$ is probably more important than the quark loop contribution.

6) The above result can also be used to make an interesting observation. If the Higgs sector is the low energy effective field theory of a NJL model with exactly the same parameters as the low energy QCD except for $M_{\pi}=0$ and $F_{\pi}=246 \mathrm{GeV}$, then we find $M_{\sigma}=$ $1915 \mathrm{GeV}$. This corresponds to $m_{\sigma}=2$ where one would expect very large deviations from the low energy behavior of scattering cross sections. Although we have not calculated these deviations the value of the width serves as an indication of their size. In a way, departure from low energy behavior will be signaled by an increasing width of the $\sigma$ to two quark decay. At $m_{\sigma} \approx 2$ the width is already fairly large.

There are some interesting issues relevant to lattice work that have not been considered in this paper. It would be important to calculate the three and four point vertices and therefore be able to calculate scattering amplitudes and their departure from low energy behavior as well as the $1 / N$ corrections to the width. It would also be interesting to study the NJL model at finite temperature and investigate the finite temperature transition in connection with the approach to the continuum chiral limit. Finally it would be important to include vector meson couplings (see, for example, [8], [11]) and confirm that for the case of Wilson fermions the vector meson masses scale appropriately and do not become of the order cutoff as the $\sigma$ particle does.

\section{Acknowledgments}

We would like to thank U.M. Heller and R. Edwards for useful discussions concerning the subject of this paper. This research was supported in part by the DOE under grant \# DE-FG05-85ER250000 and \# DE-FG05-92ER40742. 


\section{Appendix A}

Below we give the lattice constants, defined in eq. 38 and 39, that appeared in our analysis of Wilson fermions.

$r=1$

$$
\begin{aligned}
a_{0,1} & =0.0854 \\
a_{1,1} & =0.2347 \\
a_{1,2} & =0.0260 \\
a_{2,2} & =0.0597 \\
a_{2,3} & =0.0086 \\
a_{3,3} & =0.0165 \\
r_{1} & =-0.0119 \\
s_{1} & =\frac{1}{16 \pi^{2}}
\end{aligned}
$$

Although they were never used, we have also calculated the constant $R_{3}$ defined in eq. 50 and also few more of the $a_{n, m}$ 's.

$$
\begin{aligned}
R_{3} & =-0.0016 \\
a_{4,4} & =0.0050 \\
a_{4,3} & =0.0470 \\
a_{6,4} & =0.0388
\end{aligned}
$$

$r=0.1$

$$
\begin{aligned}
a_{0,1} & =0.5373 \\
a_{1,1} & =2.0721 \\
a_{1,2} & =1.3881 \\
a_{2,2} & =5.8935 \\
a_{2,3} & =6.1127 \\
a_{3,3} & =25.633 \\
r_{1} & =-0.3818 \\
s_{1} & =\frac{1}{16 \pi^{2}}
\end{aligned}
$$

The asymptotic expansions of the integrals $J_{0,1}\left(m_{q}\right)$ and $J_{1,1}\left(m_{q}\right)$ defined in eq. 37 to order $m_{q}^{2}$ are:

$$
\begin{aligned}
& J_{0,1}\left(m_{q}\right)=a_{0,1}-2 m_{q} r a_{1,2}+m_{q}^{2}\left[4 r^{2} a_{2,3}+r_{1}+s_{1}+s_{1} \log m_{q}^{2}\right]+O\left(m_{q}^{3}\right) \\
& J_{1,1}\left(m_{q}\right)=a_{1,1}-2 m_{q} r a_{2,2}+m_{q}^{2}\left[4 r^{2} a_{3,3}-a_{1,2}\right]+O\left(m_{q}^{3}\right)
\end{aligned}
$$

The leading order term of the asymptotic expansion of $J_{0,2}\left(m_{q}\right)$ defined in eq. 37 is:

$$
J_{0,2}\left(m_{q}\right)=-r_{1}-s_{1}-s_{1} \log m_{q}^{2}+O\left(m_{q}\right)
$$




\section{References}

[1] A. Dhar, R, Shankar, and S. R. Wadia, Phys. Rev. D 31, 3256 (1985).

[2] UKQCD collaboration, G.S. Bali, K. Schilling, A. Hulsebos, A.C. Irving, C. Michael, P.W. Stephenson, Phys. Lett. B 309, 378 (1993).

[3] S.P. Klevansky, Rev. Mod. Phys. 64, 649 (1992).

[4] J. Bijnens, C. Bruno and E. de Rafael, Nucl. Phys. B390, 501 (1993); S. Peris and E. de Rafael, Phys. Lett. B 309, 389 (1993); J. Bijnens, E. de Rafael and H. Zheng, preprint CERN-TH.6924/93, CTP-93/P.2917, NORDITA-93/43 N,P; J.A. Gracey, preprint LTH-314.

[5] For reviews on the subject see: H. Neuberger Nucl. Phys. B (Proc. Suppl.) 17, 17 (1990); J. Shigemitsu Nucl. Phys. B (Proc. Suppl.) 20, 515 (1991); I. Montvay Nucl. Phys. B (Proc. Suppl.) 26, 57 (1992); D. Petcher Nucl. Phys. B (Proc. Suppl.) 30, 50 (1993).

[6] A. Hasenfratz, P. Hasenfratz, K. Jansen, J. Kuti, Y. Shen, Nucl. Phys. B365, 79 (1991).

[7] W. Bardeen, C. Hill and M. Lindner, Phys. Rev. D 41, 1647 (1990).

[8] D. Ebert and M.K. Volkov, Z. Phys. C 16, 205 (1983).

[9] K.M. Bitar and P.M. Vranas, preprint FSU-SCRI-93-127, to appear in Phys. Lett. B.

[10] S. Aoki, S. Boettcher and A. Gocksch, preprint BNL-ABG-1, hep-lat/9312084.

[11] D. Ebert and H. Reinhardt, Nucl. Phys. B271, 188 (1986).

[12] M. Lüscher, P. Weisz, Nucl. Phys. B290, 25 (1987).

[13] G. Bhanot, K. Bitar, U.M. Heller, and H. Neuberger, Nucl. Phys. B343, 467 (1990).

[14] S. Duane, A.D. Kennedy, B.J. Pendleton, and D. Roweth, Phys. Lett. B 195, 216 (1987). 